\title{
On the Nature of Bifurcation in a Ratio-Dependent Predator-Prey Model with Delays
}

\author{
Changjin $X u^{1}$ and Yusen $W u^{2}$ \\ ${ }^{1}$ Guizhou Key Laboratory of Economics System Simulation, Guizhou University of Finance and Economics, Guiyang 550004, China \\ ${ }^{2}$ Department of Mathematics and Statistics, Henan University of Science and Technology, Luoyang 471003, China
}

Correspondence should be addressed to Changjin Xu; xcj403@126.com

Received 1 April 2013; Revised 29 May 2013; Accepted 2 June 2013

Academic Editor: Jinde Cao

Copyright (c) 2013 C. Xu and Y. Wu. This is an open access article distributed under the Creative Commons Attribution License, which permits unrestricted use, distribution, and reproduction in any medium, provided the original work is properly cited.

\begin{abstract}
A ratio-dependent predator-prey model with two delays is investigated. The conditions which ensure the local stability and the existence of Hopf bifurcation at the positive equilibrium of the system are obtained. It shows that the two different time delays have different effects on the dynamical behavior of the system. An example together with its numerical simulations shows the feasibility of the main results. Finally, main conclusions are included.
\end{abstract}

\section{Introduction}

After the seminal models of Volterra and Lotka in the mid1920s, understanding the dynamics of predator-prey models has been the focus of intense research in recent years. A great deal of excellent and interesting results have been reported. For example, Bhattacharyya and Mukhopadhyay [1] made a detailed discussion on the local and global dynamical behavior of an ecoepidemiological model, Kar and Ghorai [2] analyzed the local stability, global stability, influence of harvesting, and bifurcation of a delayed predator-prey model with harvesting, and Chakraborty et al. [3] studied the bifurcation and control of a bioeconomic model of a delayed prey-predator model. Bhattacharyya and Mukhopadhyay [4] focused on the spatial dynamics of nonlinear prey-predator models with prey migration and predator switching, and Chang and Wei [5] considered the bifurcation nature and optimal control of a diffusive predator-prey system with time delay and prey harvesting. For more related research, one can see [6-26].

In 2011, Wang and Pei [27] investigated the stability and Hopf bifurcation of the following delayed ratio-dependent predator-prey system:

$$
\dot{x}(t)=a x\left(t-\tau_{1}\right)-b x^{2}-\frac{c x y}{m y+x},
$$

$$
\dot{y}(t)=\frac{x\left(t-\tau_{2}\right) y\left(t-\tau_{2}\right)}{m y\left(t-\tau_{2}\right)+x\left(t-\tau_{2}\right)}-d y,
$$

where $x(t)$ and $y(t)$ represent the densities of the prey and the predator population at time $t$, respectively. The parameters $a, k, c, m, f$, and $d$ are positive constants that stand for prey intrinsic growth rate, carrying capacity, capturing rate, half capturing saturation constant, conversion rate, predator and death rate, respectively. The constants $\tau_{1} \geq 0$ and $\tau_{2} \geq$ 0 denote the time delays due to gestation of the prey and predator, respectively. For more detailed biological meaning of the coefficients of system (1), one can see [27]. Applying the Nyquist criteria and the theory of Hopf bifurcation, Wang and Pei [27] considered the stability of the positive equilibrium and the existence of the local Hopf bifurcation of system (1). By means of the center manifold and normal form theories, they obtained explicit formulae which determine the stability, direction, and other properties of bifurcating periodic solutions.

We would like to point out that Wang and Pei [27] investigated the local stability of system (1) under the assumption $\tau_{1}+\tau_{2}$ in a certain range and focused on the local Hopf bifurcation by choosing the delay $\tau_{2}$ as bifurcation parameter. A natural problem arising from this is what effect the different delays $\tau_{1}$ and $\tau_{2}$ have on the dynamical behavior of system (1). In [27], Wang and Pei did not analyze this aspect. Thus, 


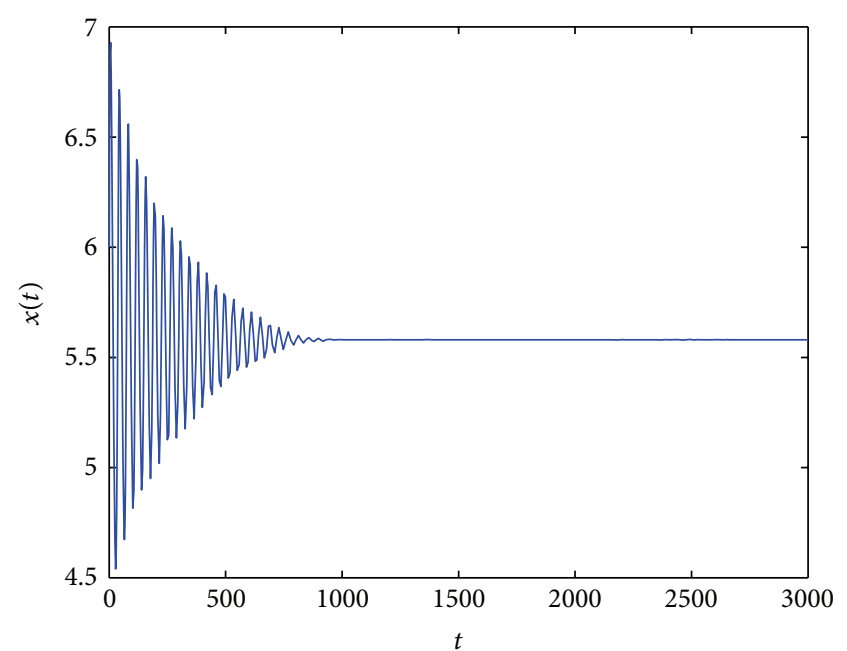

(a)

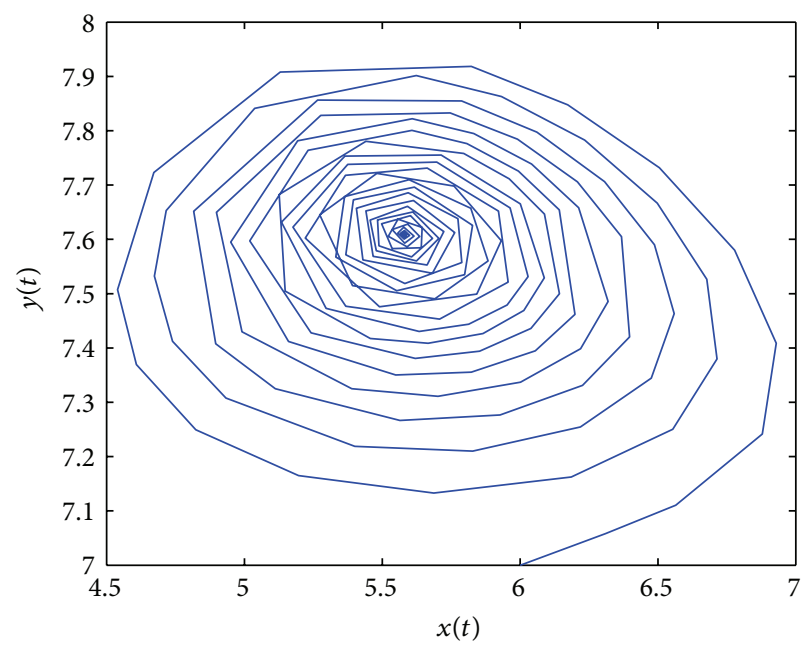

(c)

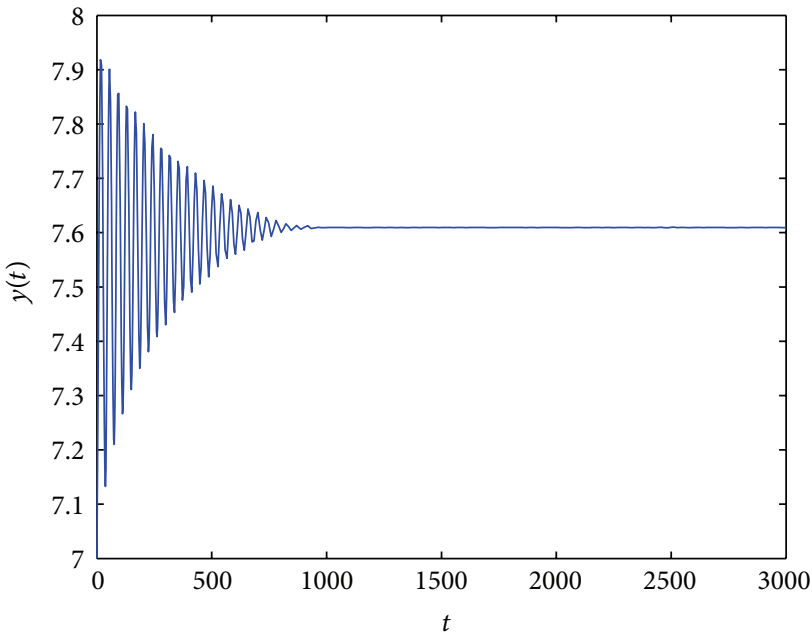

(b)

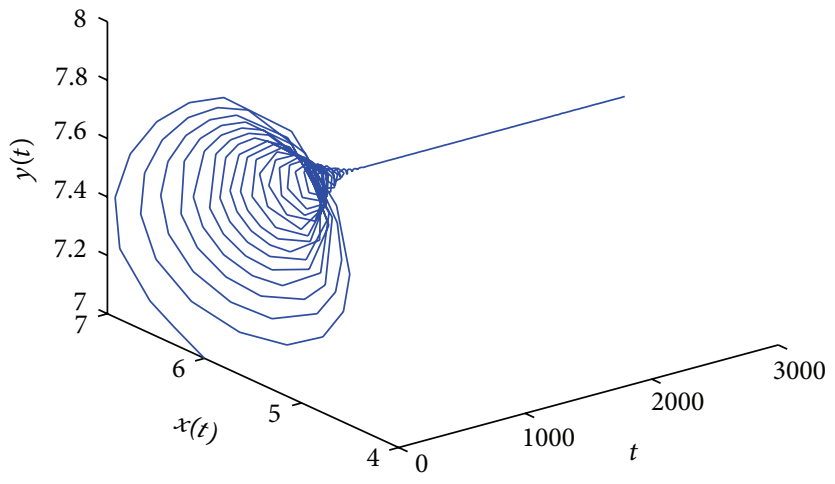

(d)

FIGURE 1: Trajectory portrait and phase portrait of system (57) with $\tau_{1}=0, \tau_{2}=2.2<\tau_{2_{0}} \approx 2.3345$. The positive equilibrium $E(5.61,7.02)$ is asymptotically stable. The initial value is $(5.6,7.45)$.

we think that it is important to deal with the effect of time delay on the dynamics of system (1). To the best of the authors knowledge, there are very few works' which deal with this topic. In this paper, we will further investigate the stability and bifurcation of model (1) as a complementarity. It will be shown that the two different time delays $\tau_{1}$ and $\tau_{2}$ have different effects on the stability and Hopf bifurcation nature of system (1).

The remainder of the paper is organized as follows. In Section 2, we investigate the stability of the positive equilibrium and the occurrence of local Hopf bifurcations. In Section 3, numerical simulations are carried out to illustrate the validity of the main results. Some main conclusions are drawn in Section 4.

\section{Stability and Local Hopf Bifurcations}

In this section, we shall study the stability of the positive equilibrium and the existence of local Hopf bifurcations.

From [27], we know that if the following conditions

(H1)

$$
\begin{aligned}
& d<f<\frac{d c}{c-m a}, \\
& c>m a \text { or } f>d, c \leq m a
\end{aligned}
$$




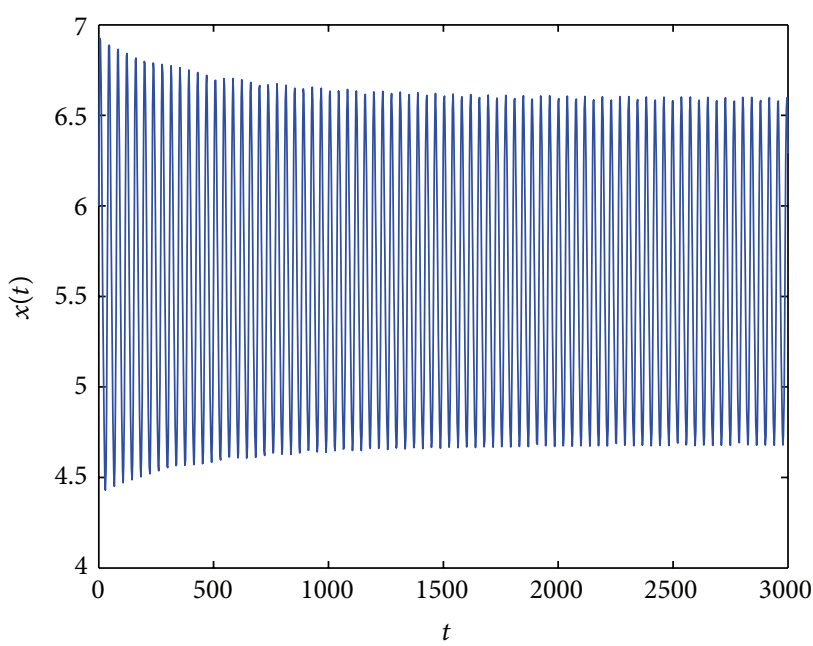

(a)

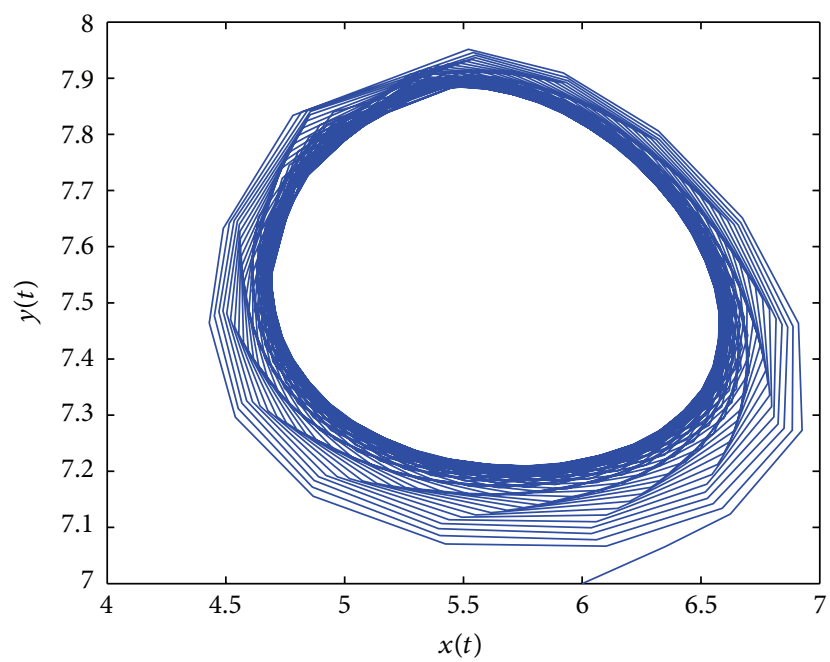

(c)

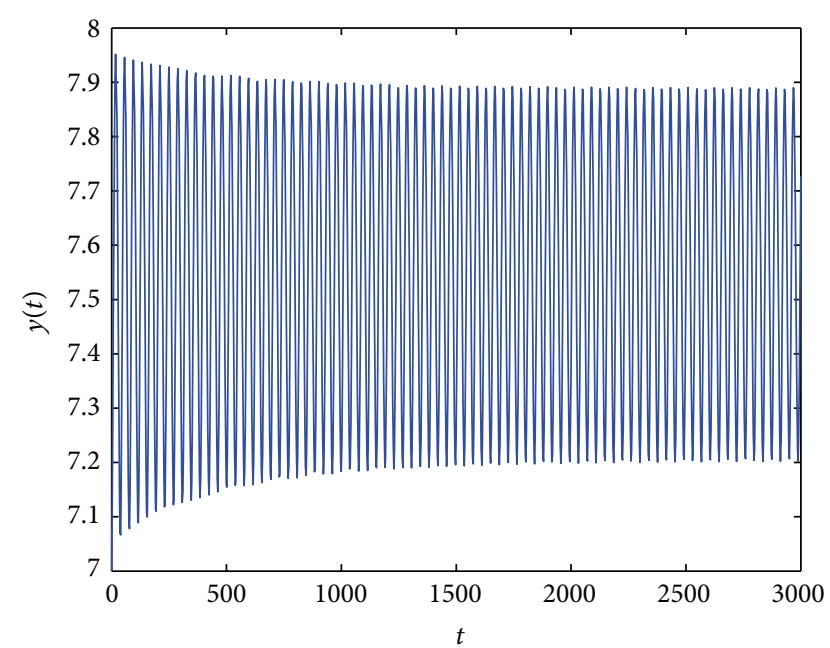

(b)

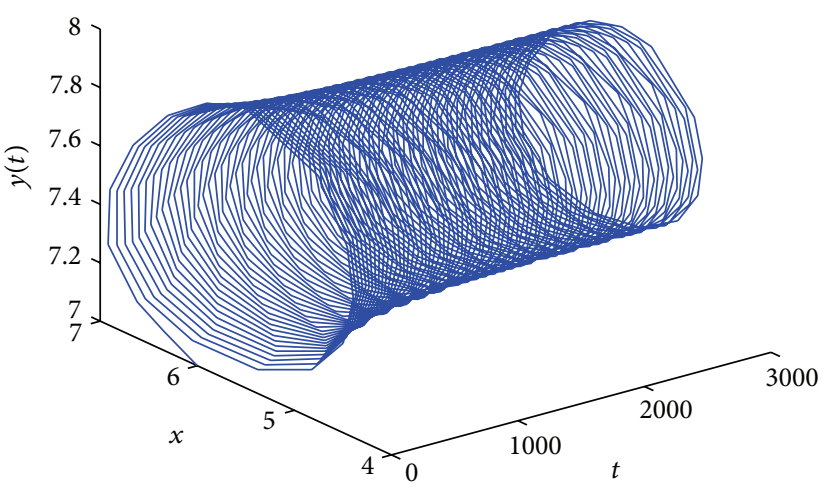

(d)

FIGURE 2: Trajectory portrait and phase portrait of system (57) with $\tau_{1}=0, \tau_{2}=2.5>\tau_{2_{0}} \approx 2.3345$. Hopf bifurcation occurs from the positive equilibrium $E(5.61,7.02)$. The initial value is $(5.6,7.45)$.

hold, then system (1) has a unique equilibrium point $E\left(x^{*}\right.$, $\left.y^{*}\right)$, where

$$
x^{*}=\frac{a k l-b_{1}(k+l)}{a k l}, \quad y^{*}=\frac{b_{1}}{k l} .
$$

Let $\bar{x}(t)=x(t)-x^{*}$ and $\bar{y}(t)=y(t)-y^{*}$ and still denote $\bar{x}(t), \bar{y}(t)$ by $x(t), y(t)$, respectively. Then (1) reads as

$$
\begin{gathered}
\dot{x}(t)=p_{1} x+p_{2} y+p_{3} x\left(t-\tau_{1}\right), \\
\dot{y}(t)=p_{4} y+p_{5} x\left(t-\tau_{2}\right)+p_{6} y\left(t-\tau_{2}\right),
\end{gathered}
$$

where

$$
\begin{gathered}
p_{1}=\frac{c\left(f^{2}-d^{2}\right)-2 m a f^{2}}{m f^{2}}, \\
p_{2}=-\frac{d^{2} c}{f^{2}},
\end{gathered}
$$

$$
\begin{gathered}
p_{3}=a, \\
p_{4}=-d, \\
p_{5}=\frac{(f-d)^{2}}{m f}, \\
p_{6}=\frac{d^{2}}{f} .
\end{gathered}
$$

The characteristic equation of (4) is given by

$$
\operatorname{det}\left(\begin{array}{cc}
\lambda-p_{1}-p_{3} e^{-\lambda \tau_{1}} & -p_{2} \\
-p_{5} e^{-\lambda \tau_{2}} & \lambda-p_{4}-p_{6} e^{-\lambda \tau_{2}}
\end{array}\right)=0
$$




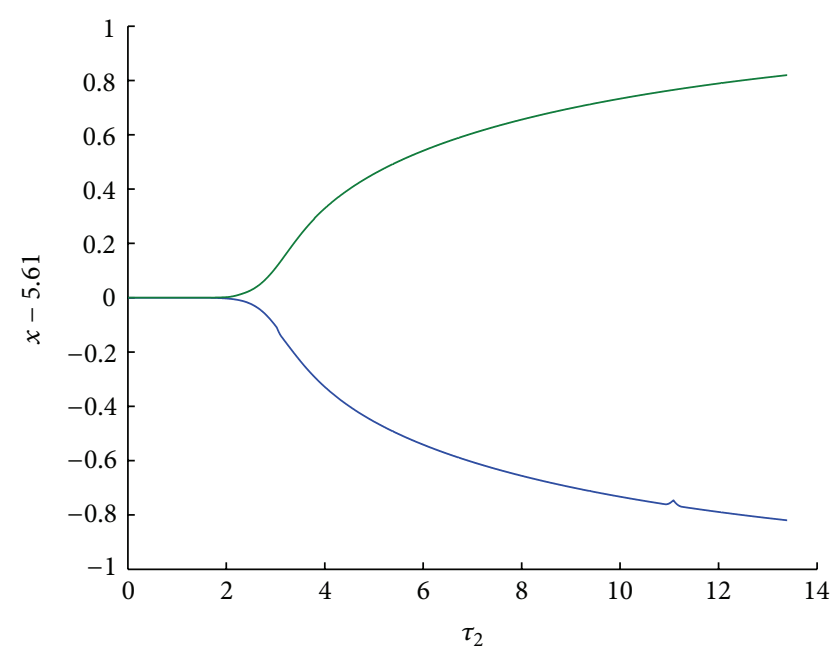

FIGURE 3: Bifurcation diagram with respect to the time delay $\tau_{2}$ for system (57) with $\tau_{1}=0$.

That is,

$$
\begin{aligned}
\lambda^{2}- & \left(p_{1}+p_{4}\right) \lambda-p_{3} \lambda e^{-\lambda \tau_{1}}-p_{6} \lambda e^{-\lambda \tau_{2}}+p_{3} p_{4} e^{-\lambda \tau_{1}} \\
& +\left(p_{1} p_{6}-p_{2} p_{5}\right) e^{-\lambda \tau_{2}}+p_{3} p_{6} e^{-\lambda\left(\tau_{1}+\tau_{2}\right)}+p_{1} p_{4}=0 .
\end{aligned}
$$

The following lemma is important for us to analyze the distribution of roots of the transcendental equation (7).

Lemma 1 (see [28]). For the transcendental equation

$$
\begin{aligned}
P\left(\lambda, e^{-\lambda \tau_{1}}, \ldots, e^{-\lambda \tau_{m}}\right) & \\
= & \lambda^{n}+p_{1}^{(0)} \lambda^{n-1}+\cdots+p_{n-1}^{(0)} \lambda+p_{n}^{(0)} \\
& +\left[p_{1}^{(1)} \lambda^{n-1}+\cdots+p_{n-1}^{(1)} \lambda+p_{n}^{(1)}\right] e^{-\lambda \tau_{1}} \\
& +\cdots+\left[p_{1}^{(m)} \lambda^{n-1}+\cdots+p_{n-1}^{(m)} \lambda+p_{n}^{(m)}\right] e^{-\lambda \tau_{m}}=0,
\end{aligned}
$$

as $\left(\tau_{1}, \tau_{2}, \tau_{3}, \ldots, \tau_{m}\right)$ vary, the sum of orders of the zeros of $P\left(\lambda, e^{-\lambda \tau_{1}}, \ldots, e^{-\lambda \tau_{m}}\right)$ in the open right half plane can change, and only a zero appears on or crosses the imaginary axis.

In the sequel, we consider five cases.
Case 1. $\tau_{1}=\tau_{2}=0$. Equation (7) becomes

$$
\begin{aligned}
\lambda^{2}- & \left(p_{1}+p_{3}+p_{4}+p_{6}\right) \lambda^{2} \\
& +\left(p_{3} p_{4}+p_{1} p_{4}+p_{1} p_{6}-p_{2} p_{5}+p_{3} p_{6}\right)=0 .
\end{aligned}
$$

All roots of (9) have a negative real part if the following condition holds:

$(\mathrm{H} 2)$

$$
\begin{gathered}
p_{1}+p_{3}+p_{4}+p_{6}<0 \\
p_{3} p_{4}+p_{1} p_{4}+p_{1} p_{6}-p_{2} p_{5}+p_{3} p_{6}>0 .
\end{gathered}
$$

Then the equilibrium point $E\left(x^{*}, y^{*}\right)$ is locally asymptotically stable when the conditions (H1) and (H2) are satisfied.

Case 2. $\tau_{1}=0, \tau_{2}>0$. Equation (7) becomes

$$
\lambda^{2}-\left(p_{1}+p_{4}\right) \lambda+p_{3} p_{4}+p_{1} p_{4}
$$

$$
-\left(p_{6} \lambda+p_{2} p_{5}-p_{1} p_{6}-p_{3} p_{6}\right) e^{-\lambda \tau_{2}}=0 \text {. }
$$

For $\omega>0$, let $i \omega$ be a root of (11). Then it follows that

$$
\begin{gathered}
\left(p_{1} p_{6}-p_{2} p_{5}+p_{3} p_{6}\right) \cos \omega \tau_{2}-p_{6} \omega \sin \omega \tau_{2} \\
=\omega^{2}-p_{3} p_{4}-p_{1} p_{4} \\
p_{6} \omega \cos \omega \tau_{2}+\left(p_{1} p_{6}-p_{2} p_{5}+p_{3} p_{6}\right) \sin \omega \tau_{2} \\
=-\left(p_{1}+p_{3}+p_{4}\right) \omega
\end{gathered}
$$

which is equivalent to

$$
\omega^{4}+r_{1} \omega^{2}+r_{2}=0
$$

where

$$
\begin{aligned}
& r_{1}=\left(p_{1}+p_{3}+p_{4}\right)^{2}-2\left(p_{3} p_{4}-p_{1} p_{4}\right)-p_{6}^{2}, \\
& r_{2}=\left(p_{3} p_{4}-p_{1} p_{4}\right)^{2}-\left(p_{1} p_{6}-p_{2} p_{5}+p_{3} p_{6}\right)^{2} .
\end{aligned}
$$

Define $\Delta_{1}=r_{1}^{2}-4 r_{2}$. Following Cao and Xiao [29] and Theorem 2.1 in Ge and Yan [30], we have the following result.

Lemma 2. If (HI) holds, then

(i) if $r_{1}<0$ and $\Delta_{1}=0$, then (11) with $\tau_{2}=\tau_{2_{n}}^{+}$has a pair of pure imaginary roots $\pm i \omega_{+}$;

(ii) if $r_{1}<0$ and $\Delta_{1}>0$, then (11) with $\tau_{2}=\tau_{2_{n}}^{+}$has two pairs of pure imaginary roots $\pm i \omega_{+}$and $\pm i \omega_{-}$, where

$$
\tau_{2_{n}}^{ \pm}=\frac{1}{\omega_{ \pm}} \arccos \left[\frac{\left(\omega_{ \pm}^{2}-p_{3} p_{4}-p_{1} p_{4}\right)\left(p_{1} p_{6}-p_{2} p_{5}+p_{3} p_{6}\right)-p_{6}\left(p_{1}+p_{3}+p_{4}\right) \omega_{ \pm}^{2}}{\left(p_{1} p_{6}-p_{2} p_{5}+p_{3} p_{6}\right)^{2}+\left(p_{6} \omega_{ \pm}\right)^{2}}\right]+\frac{2 n \pi}{\omega_{ \pm}}
$$




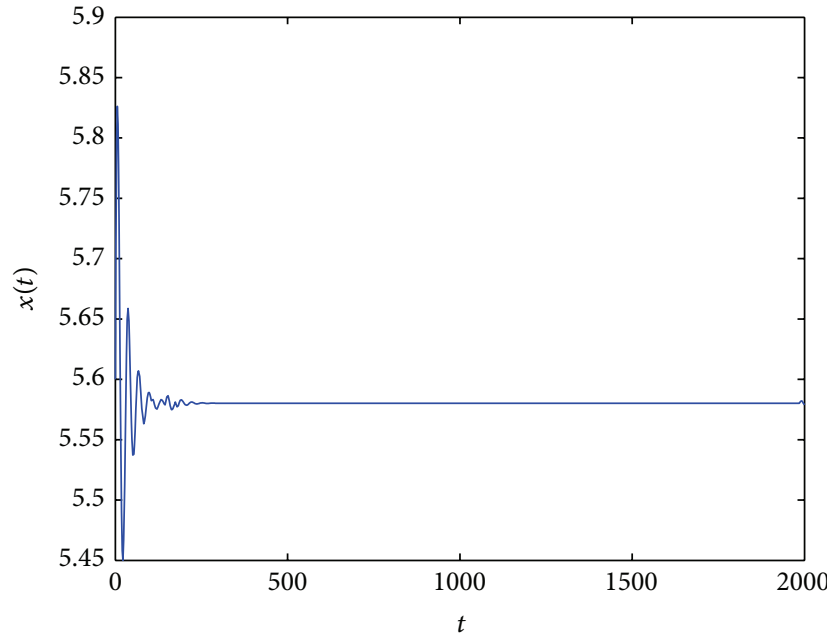

(a)

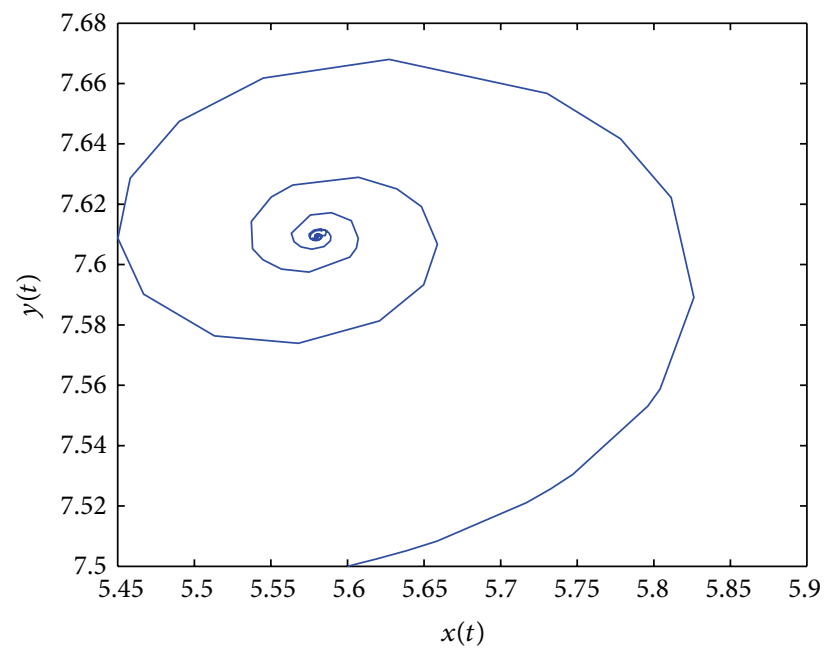

(c)

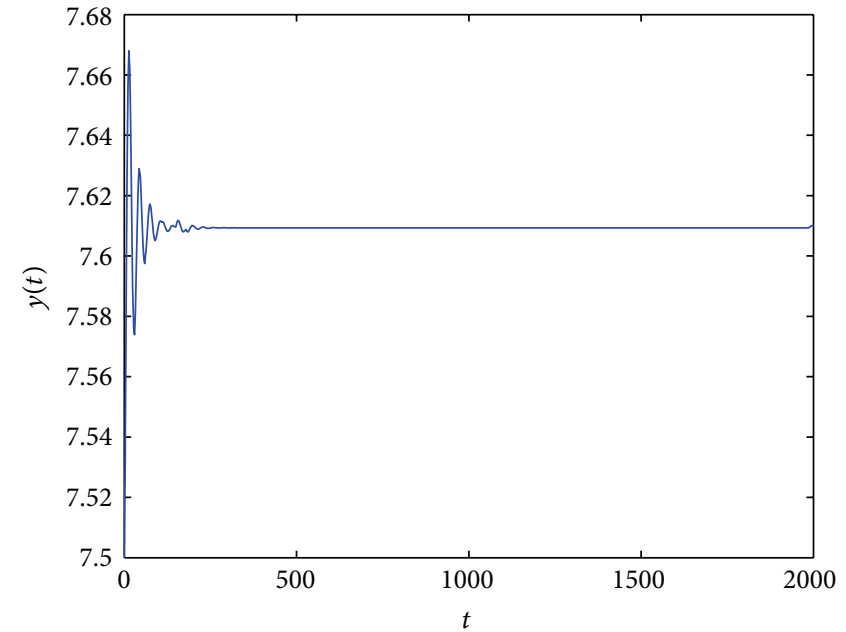

(b)

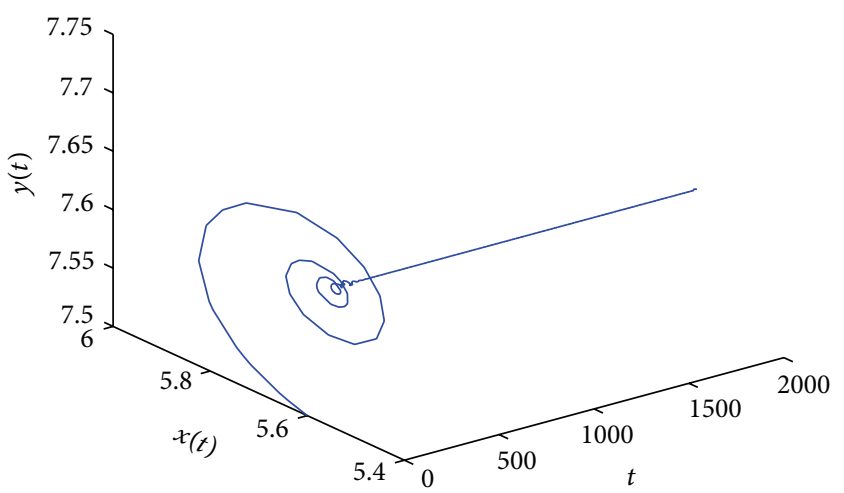

(d)

FIGURE 4: Trajectory portrait and phase portrait of system (57) with $\tau_{2}=1.8, \tau_{1}=0.75<\tau_{1_{0}} \approx 0.8013$. The positive equilibrium $E(5.61,7.02)$ is asymptotically stable. The initial value is $(5.6,7.45)$.

and $\omega_{ \pm}$satisfies

$$
\omega_{+}^{2}=\frac{-r_{1}+\sqrt{\Delta_{1}}}{2}, \quad \omega_{-}^{2}=\frac{-r_{1}-\sqrt{\Delta_{1}}}{2}
$$

(iii) if $r_{1}>0$ or $\Delta_{1}<0$, then all the roots of (11) have negative real parts for $\tau_{2} \geq 0$. From Lemma 2, one has the following result.

Theorem 3. Let $\tau_{2_{n}}^{ \pm}$be defined by (15). Under the condition (H1),

(i) if $r_{1}<0$ and $\Delta_{1}=0$, then the trivial solution $\left(x_{*}, y_{*}\right)^{T}$ of (11) is asymptotically stable for all $\tau_{2} \in\left[0, \tau_{2_{0}}\right)$ and unstable for $\tau_{2}>\tau_{2_{0}}$. That is, Hopf bifurcation occurs when $\tau_{2}=\tau_{2_{0}}$; (ii) if $r_{1}>0$ or $\Delta_{1}<0$, then there are Hopf bifurcations near the trivial solution $\left(x_{*}, y_{*}\right)^{T}$ of (11) when $\tau_{2}=\tau_{2_{n}}^{+}$ and $\tau_{2}=\tau_{2_{n}}^{-}$.

Case 3. $\tau_{1}>0, \tau_{2}=0$. Equation (7) takes the form

$$
\begin{gathered}
\lambda^{2}-\left(p_{1}+p_{3}+p_{4}+p_{6}\right) \lambda+p_{1} p_{6}-p_{2} p_{5}+p_{1} p_{4} \\
-\left(p_{3} \lambda-p_{3} p_{4}-p_{3} p_{6}\right) e^{-\lambda \tau_{1}}=0
\end{gathered}
$$

For $\eta>0$, let $i \eta$ be a root of (17). Then it follows that

$$
\begin{gathered}
\left(p_{3} p_{4}+p_{3} p_{6}\right) \cos \eta \tau_{1}-p_{3} \eta \sin \eta \tau_{1} \\
=\eta^{2}+p_{2} p_{5}-p_{1} p_{6}-p_{1} p_{4}
\end{gathered}
$$




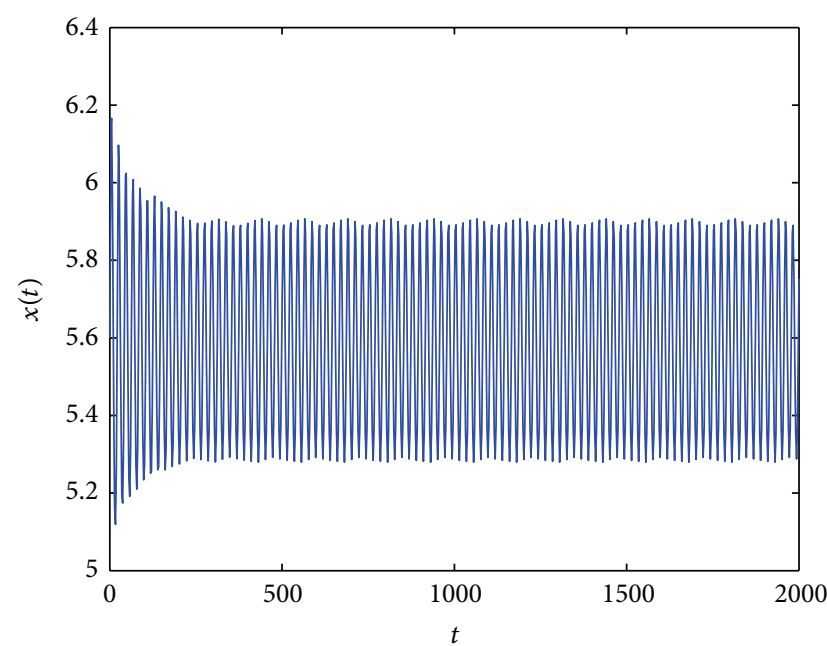

(a)

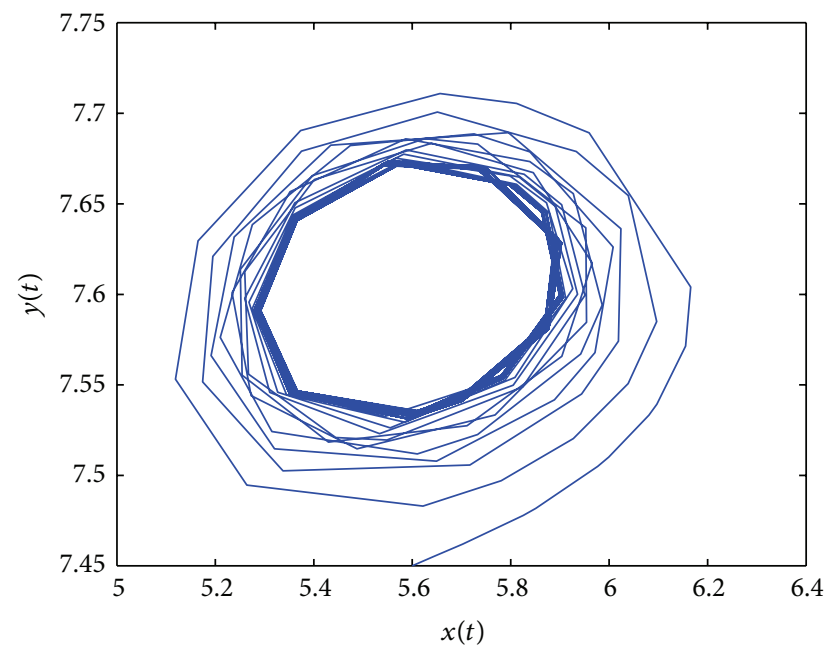

(c)

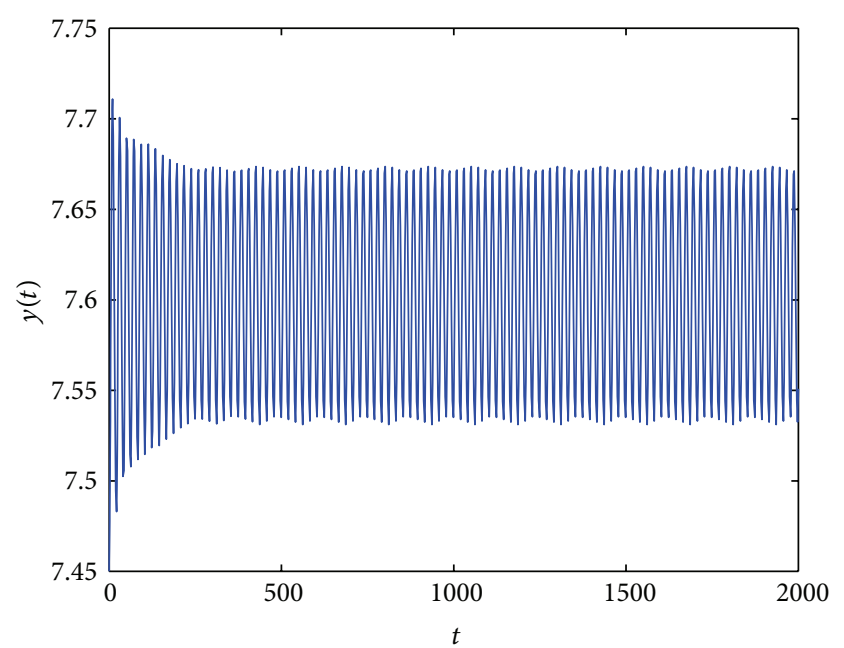

(b)

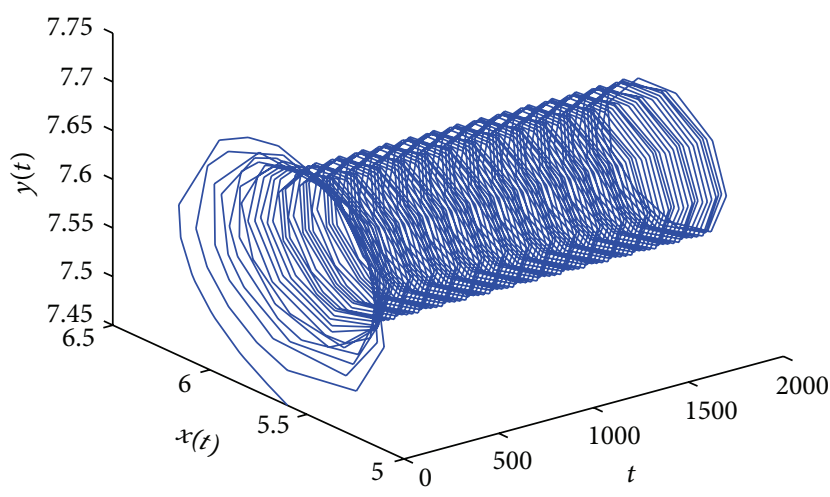

(d)

FIGURE 5: Trajectory portrait and phase portrait of system (57) with $\tau_{2}=1.8, \tau_{1}=0.98>\tau_{1_{0}} \approx 0.8013$. Hopf bifurcation occurs from the positive equilibrium $E(5.61,7.02)$. The initial value is $(5.6,7.45)$.

$$
\begin{gathered}
p_{3} \eta \cos \eta \tau_{1}+\left(p_{3} p_{4}+p_{3} p_{6}\right) \sin \eta \tau_{1} \\
=-\left(p_{1}+p_{4}+p_{6}\right)
\end{gathered}
$$

which is equivalent to

$$
\eta^{4}+s_{1} \eta^{2}+s_{2}=0
$$

where

$$
\begin{aligned}
& s_{1}=\left(p_{1}+p_{4}+p_{6}\right)^{2}+2\left(p_{2} p_{5}-p_{1} p_{6}-p_{1} p_{4}\right)-p_{3}^{2}, \\
& s_{2}=\left(p_{2} p_{5}-p_{1} p_{6}-p_{1} p_{4}\right)^{2}-\left(p_{3} p_{4}+p_{3} p_{6}\right)^{2} .
\end{aligned}
$$

Define $\Delta_{2}=s_{1}^{2}-4 s_{2}$. Following the Theorem 2.1 in Ge and Yan [30], we have the following result.

Lemma 4. If $(\mathrm{H} 1)$ holds, then

(i) if $s_{1}<0$ and $\Delta_{2}=0$, then (17) with $\tau_{1}=\tau_{1_{n}}^{+}$has a pair of pure imaginary roots \pm in ;

(ii) if $s_{1}<0$ and $\Delta_{2}>0$, then (17) with $\tau_{1}=\tau_{1_{n}}^{+}$has two pairs of pure imaginary roots $\pm i \eta_{+}$and $\pm i \eta_{-}$, where

$$
\tau_{1_{n}}^{ \pm}=\frac{1}{\eta_{ \pm}} \arccos \left[\frac{\left(\eta_{ \pm}^{2}+p_{2} p_{5}-p_{1} p_{6}-p_{1} p_{4}\right)\left(p_{3} p_{4}+p_{3} p_{6}\right)-p_{3}\left(p_{1}+p_{4}+p_{6}\right) \eta_{ \pm}}{\left(p_{3} p_{4}+p_{3} p_{6}\right)^{2}+\left(p_{3} \eta_{ \pm}\right)^{2}}\right]+\frac{2 n \pi}{\eta_{ \pm}}
$$




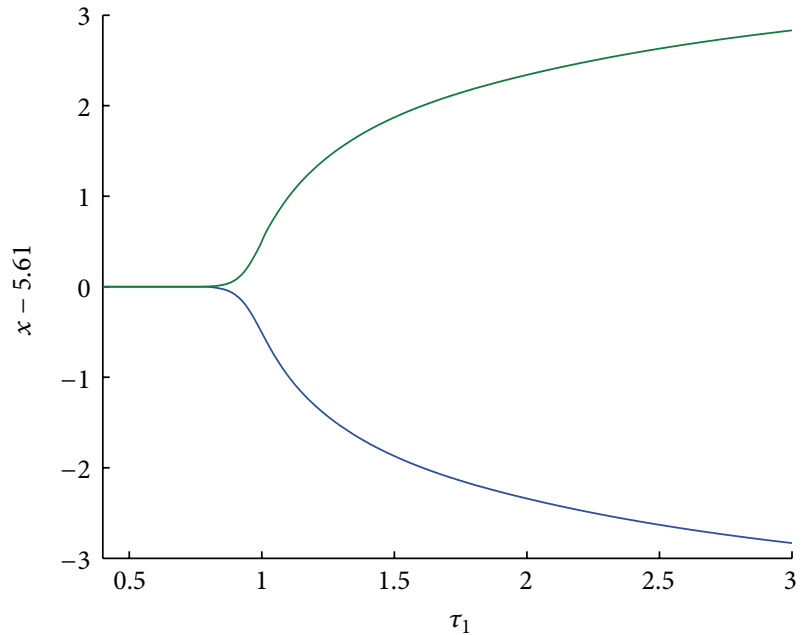

FIGURE 6: Bifurcation diagram with respect to the time delay $\tau_{1}$ for system (57) with $\tau_{2}=1.8$.

and $\eta_{ \pm}$satisfies

$$
\eta_{+}^{2}=\frac{-s_{1}+\sqrt{\Delta_{2}}}{2}, \quad \eta_{-}^{2}=\frac{-s_{1}-\sqrt{\Delta_{2}}}{2}
$$

(iii) if $s_{1}>0$ or $\Delta_{2}<0$, then all the roots of (17) have negative real parts for $\tau_{1} \geq 0$.

From Lemma 4, we have the following result.

Theorem 5. Let $\tau_{1_{n}}^{ \pm}$be defined by (21). Under the condition (H1),

(i) if $s_{1}<0$ and $\Delta_{2}=0$, then the trivial solution $\left(x_{*}, y_{*}\right)^{T}$ of (17) is asymptotically stable for all $\tau_{1} \in\left[0, \tau_{1_{0}}\right)$ and unstable for $\tau_{1}>\tau_{1_{0}}$. That is, Hopf bifurcation occurs when $\tau_{1}=\tau_{1_{0}}$;

(ii) if $s_{1}>0$ or $\Delta_{2}<0$, then there are Hopf bifurcations near the trivial solution $\left(x_{*}, y_{*}\right)^{T}$ of $(17)$ when $\tau_{1}=\tau_{1_{n}}^{+}$ and $\tau_{1}=\tau_{1_{n}}^{-}$.

Case 4. $\tau_{1}>0, \tau_{2}>0$. We consider (7) with $\tau_{2}$ in its stable interval, by regarding $\tau_{1}$ as a parameter. Without loss of generality, we consider system (1) under the assumptions (H1) and (H2). Let $i \eta^{*}\left(\eta^{*}>0\right)$ be a root of (7). Then we can obtain

$$
\eta^{* 4}+k_{1} \eta^{* 3}+k_{2} \eta^{* 2}+k_{3} \eta^{*}+k_{4}=0
$$

where

$$
\begin{aligned}
& k_{1}=2 p_{6} \sin \eta^{*} \tau_{2}, \\
& k_{2}=p_{6}^{2} \sin ^{2} \eta^{*} \tau_{2}-2\left(p_{1} p_{6}-p_{2} p_{5}\right) \cos \eta^{*} \tau_{2}
\end{aligned}
$$

$$
\begin{aligned}
& +\left(p-1+p_{4}+p_{6} \cos \eta^{*} \tau_{2}\right)^{2}, \\
k_{3}= & -2 p_{6}\left(p_{1} p_{6}-p_{2} p_{5}\right) \sin \eta^{*} \tau_{2} \cos \eta^{*} \tau_{2}, \\
k_{4}= & p_{6}\left(p_{1} p_{6}-p_{2} p_{5}\right)^{2}+\left(p_{1} p_{6}-p_{2} p_{5}\right)^{2} \sin ^{2} \eta^{*} \tau_{2} \\
& +2\left(p_{1}+p_{4}+p_{6} \cos \eta^{*} \tau_{2}\right)\left(p_{1} p_{6}-p_{2} p_{5}\right) \sin \eta^{*} \tau_{2} \\
& -\left(p_{3}+p_{4}+p_{3} p_{6} \cos \eta^{*} \tau_{2}\right)^{2}-\left(p_{3} \eta+p_{3} p_{6} \sin \eta^{*} \tau_{2}\right)^{2} .
\end{aligned}
$$

Denote

$$
H\left(\eta^{*}\right)=\eta^{* 4}+k_{1} \eta^{* 3}+k_{2} \eta^{* 2}+k_{3} \eta^{*}+k_{4} .
$$

Assume that

(H3)

$$
k_{4}<0
$$

It is easy to check that $H(0)<0$ if (H5) holds and $\lim _{\eta^{*} \rightarrow+\infty} H\left(\eta^{*}\right)=+\infty$. We can obtain that (25) has finite positive roots $\eta_{1}^{*}, \eta_{2}^{*}, \ldots, \eta_{n}^{*}$. For every fixed $\eta_{i}^{*}, i=$ $1,2,3, \ldots, k$, there exists a sequence $\left\{\tau_{1_{i}}^{j} \mid j=1,2,3, \ldots\right\}$, such that (25) holds. Let

$$
\tau_{1_{0}}=\min \left\{\tau_{1_{i}}^{j} \mid i=1,2, \ldots, k ; j=1,2, \ldots\right\} .
$$

When $\tau_{1}=\tau_{1_{0}}$, (7) has a pair of purely imaginary roots $\pm i \widetilde{\eta}^{*}$ for $\tau_{2} \in\left[0, \tau_{2_{0}}\right)$.

In the following, we assume that

(H4)

$$
\left[\frac{d(\operatorname{Re} \lambda)}{d \tau_{1}}\right]_{\lambda=i \tilde{\eta}^{*}} \neq 0 .
$$

Thus, by the general Hopf bifurcation theorem for FDEs in Hale [31], we have the following result on the stability and Hopf bifurcation in system (1).

Theorem 6. For system (1), suppose that (H1), (H2), (H3), and (H4) are satisfied and $\tau_{2} \in\left[0, \tau_{2_{0}}\right)$. Then the positive equilibrium $E\left(x^{*}, y^{*}\right)$ is asymptotically stable when $\tau_{1} \in$ $\left[0, \tau_{1_{0}}\right)$, and system (1) undergoes a Hopf bifurcation at the positive equilibrium $E\left(x^{*}, y^{*}\right)$ when $\tau_{1}=\tau_{1_{0}}$.

Case 5. $\tau_{1}>0, \tau_{2}>0$. We consider (7) with $\tau_{1}$ in its stable interval, by regarding $\tau_{2}$ as a parameter. Without loss of generality, we consider system (1) under the assumptions (H1) and (H2). Let $i \omega^{*}\left(\omega^{*}>0\right)$ be a root of (7). Then we can obtain

$$
\omega^{* 4}+t_{1} \omega^{* 3}+t_{2} \omega^{* 2}+t_{3} \omega^{*}+t_{4}=0,
$$

where

$$
\begin{aligned}
t_{1}= & 2 p_{3} \sin \omega^{*} \tau_{1}, \\
t_{2}= & p_{3}^{2} \sin ^{2} \omega^{*} \tau_{1}-2 p_{3} p_{4} \cos \omega^{*} \tau_{1} \\
& -\left(p_{1}+p_{4}+p_{3} \cos \omega^{*} \tau_{1}\right)^{2}, \\
t_{3}= & 2 p_{3} p_{4}\left(p_{1}+p_{4}+p_{3} \sin \omega^{*} \tau_{1}\right) \sin \omega^{*} \tau_{1}
\end{aligned}
$$




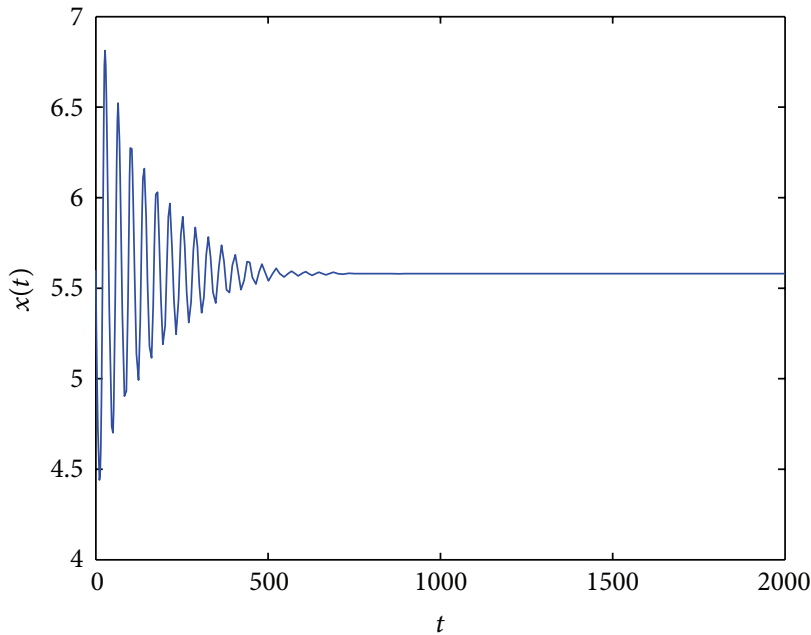

(a)

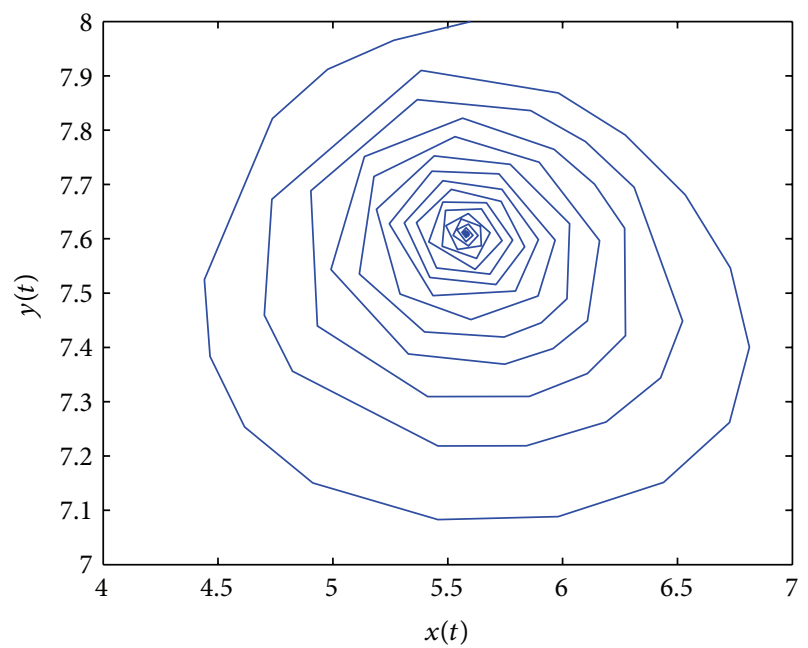

(c)

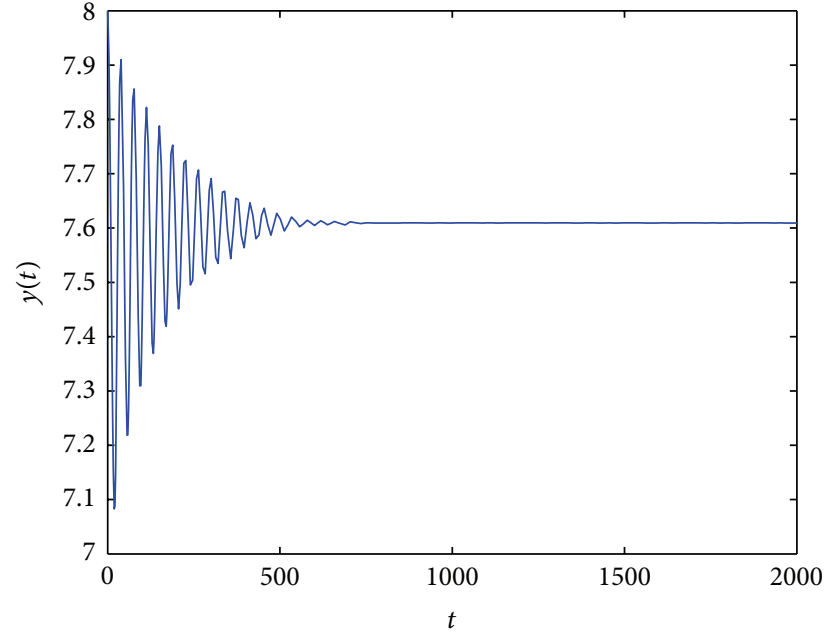

(b)

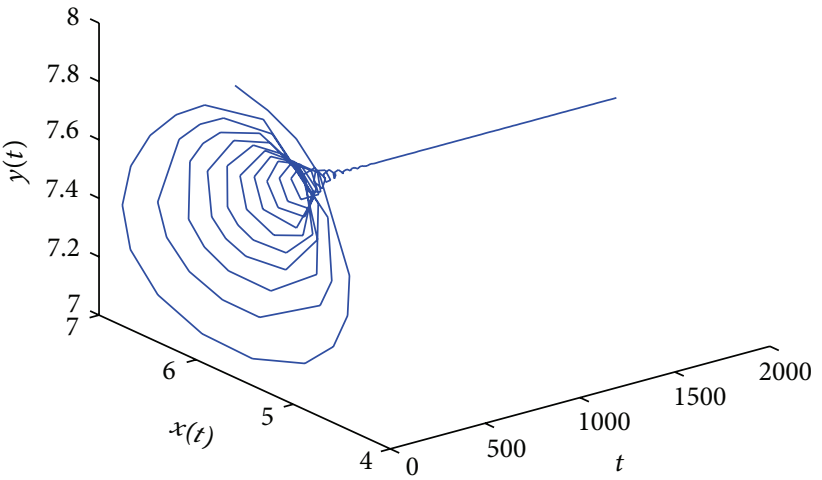

(d)

FIGURE 7: Trajectory portrait and phase portrait of system (57) with $\tau_{2}=0, \tau_{1}=0.90<\tau_{1_{0}} \approx 0.9122$. The positive equilibrium $E(5.61,7.02)$ is asymptotically stable. The initial value is $(5.6,8)$.

$$
\begin{aligned}
& -2 p_{3}^{2} p_{4} \sin \omega^{*} \tau_{1} \cos \omega^{*} \tau_{1} \\
t_{4}= & \left(p_{3} p_{4}\right)^{2} \sin ^{2} \omega^{*} \tau_{2}-\left(p_{1} p_{6}-p_{2} p_{5}+p_{3} p_{6} \cos \omega^{*} \tau_{1}\right)^{2} \\
& -\left(p_{6} \omega^{*}+p_{3} p_{6} \sin \omega^{*} \tau_{1}\right)^{2}
\end{aligned}
$$

Denote

$$
H_{*}\left(\omega^{*}\right)=\omega^{* 4}+t_{1} \omega^{* 3}+t_{2} \omega^{* 2}+t_{3} \omega^{*}+t_{4}
$$

Obviously, $H(0)<0$ if (H5) holds and $\lim _{\omega^{*} \rightarrow+\infty} H_{*}\left(\omega^{*}\right)=+\infty$. We can obtain that (31) has finite positive roots $\omega_{1}^{*}, \omega_{2}^{*}, \ldots, \omega_{n}^{*}$. For every fixed $\omega_{i}^{*}$, $i=1,2,3, \ldots, k$, there exists a sequence $\left\{\tau_{2_{i}}^{j} \mid j=1,2,3, \ldots\right\}$, such that (31) holds. Let

$$
\tau_{2_{0}}=\min \left\{\tau_{2_{i}}^{j} \mid i=1,2, \ldots, k ; j=1,2, \ldots\right\} .
$$

When $\tau_{2}=\tau_{2_{0}}$, (7) has a pair of purely imaginary roots $\pm i \overline{\omega^{*}}$ for $\tau_{1} \in\left[0, \tau_{1_{0}}\right)$.

In the following, we assume that

(H5)

$$
\left[\frac{d(\operatorname{Re} \lambda)}{d \tau_{2}}\right]_{\lambda=i \overline{\omega^{*}}} \neq 0
$$

In view of the general Hopf bifurcation theorem for FDEs in Hale [31], we have the following result on the stability and Hopf bifurcation in system (1).

Theorem 7. For system (1), assume that (H1), (H2), (H3), and (H5) are satisfied and $\tau_{1} \in\left[0, \tau_{1_{0}}\right)$. Then the positive equilibrium $E\left(x^{*}, y^{*}\right)$ is asymptotically stable when $\tau_{2} \in$ $\left[0, \tau_{2_{0}}\right)$, and system (1) undergoes a Hopf bifurcation at the positive equilibrium $E\left(x^{*}, y^{*}\right)$ when $\tau_{2}=\tau_{2_{0}}$. 


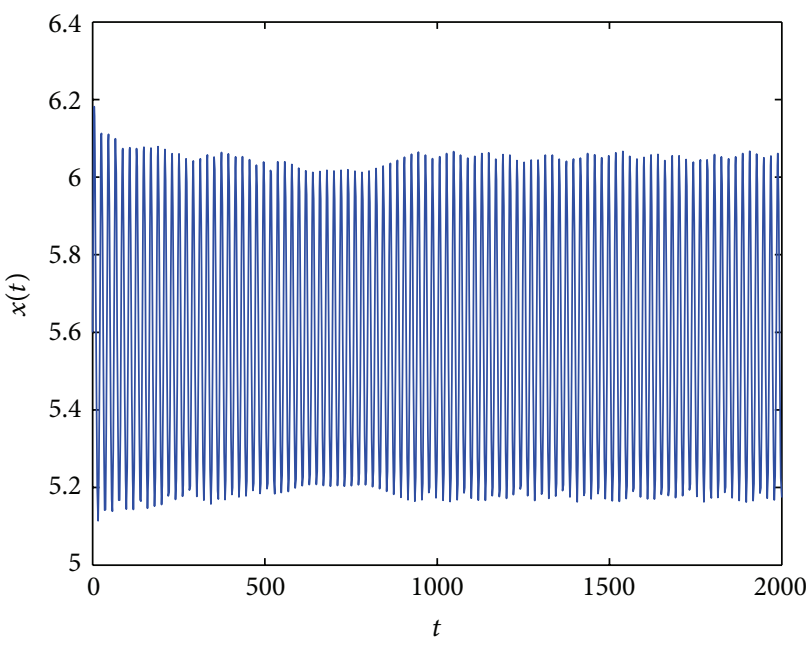

(a)

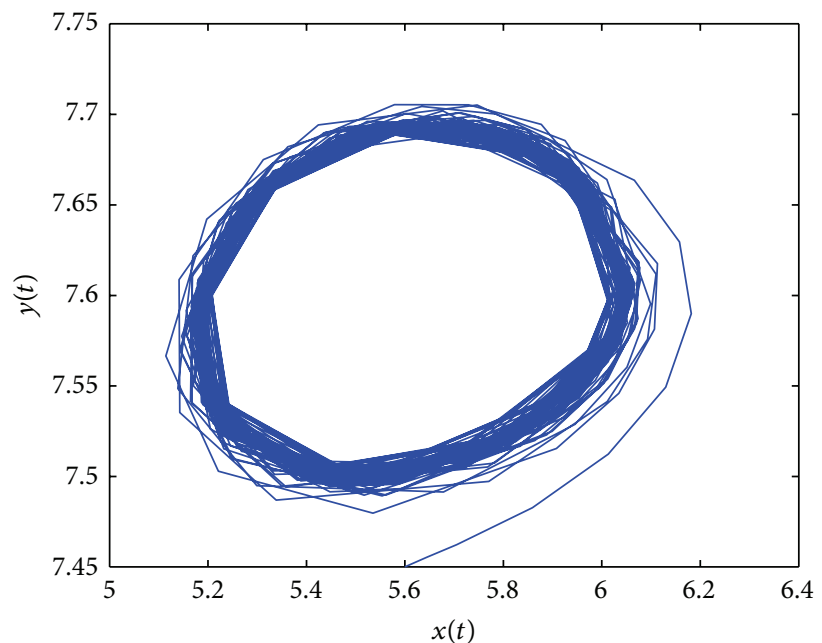

(c)

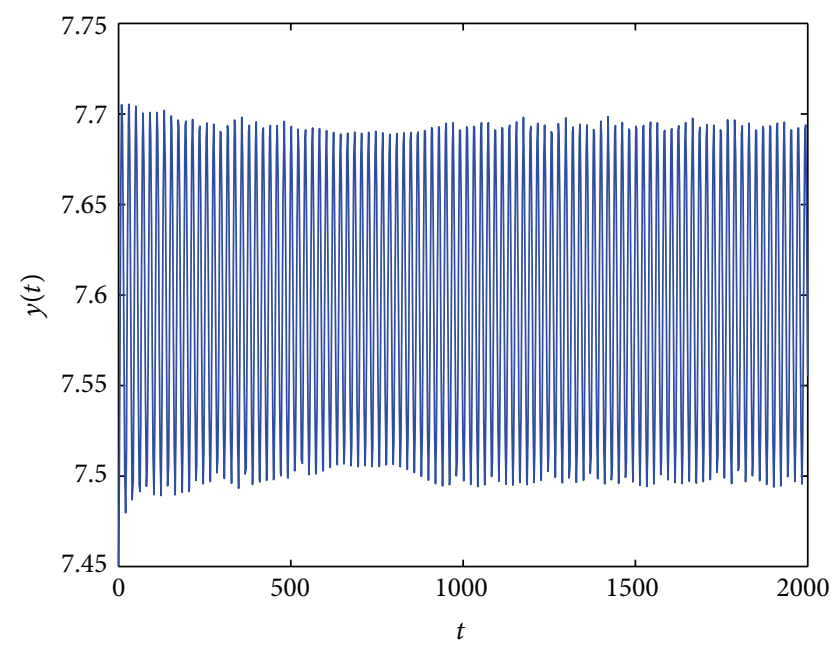

(b)

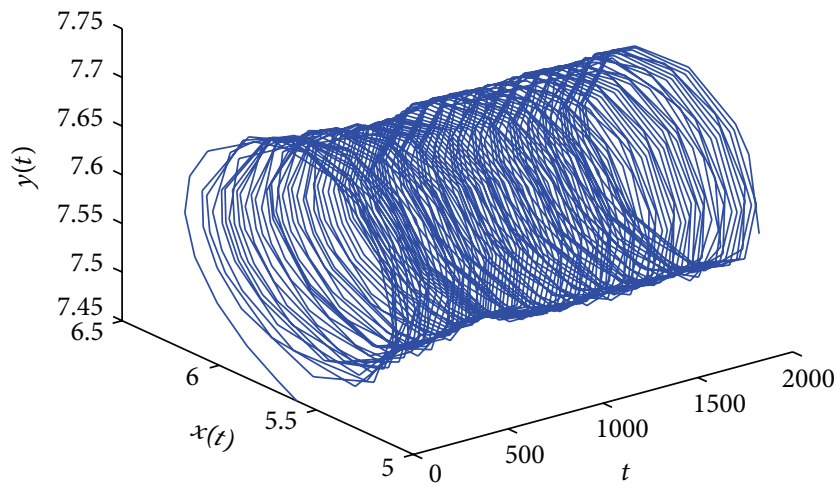

(d)

FIGURE 8: Trajectory portrait and phase portrait of system (57) with $\tau_{2}=0, \tau_{1}=1.0>\tau_{1_{0}} \approx 0.9122$. Hopf bifurcation occurs from the positive equilibrium $E_{0}(5.61,7.02)$. The initial value is $(5.6,7.45)$.

Case 6. $\tau_{1}=\tau_{2}=\tau$. Equation (7) becomes

$$
\begin{aligned}
\lambda^{2}- & \left(p_{1}+p_{4}\right) \lambda+p_{1} p_{4} \\
& -\left[\left(p_{2}+p_{6}\right) \lambda-\left(p-3 p_{4}+p_{1} p_{6}-p_{2} p_{5}\right)\right] e^{-\lambda \tau} \\
& +p_{3} p_{6} \lambda e^{-2 \lambda \tau}=0,
\end{aligned}
$$

which is equivalent to

$$
\left[\lambda^{2}-\left(p_{1}+p_{4}\right) \lambda+p_{1} p_{4}\right] e^{\lambda \tau}
$$

$$
\begin{aligned}
& -\left[\left(p_{2}+p_{6}\right) \lambda-\left(p_{3} p_{4}+p_{1} p_{6}-p_{2} p_{5}\right)\right] \\
& +p_{3} p_{6} \lambda e^{-\lambda \tau}=0 .
\end{aligned}
$$

When $\tau=0$, (35) becomes

$$
\begin{aligned}
\lambda^{2}- & \left(p_{1}+p_{3}+p_{4}+p_{6}\right) \lambda^{2} \\
& +\left(p_{1} p_{4}+p_{3} p_{4}+p_{1} p_{6}-p_{2} p_{5}+p_{3} p_{6}\right)=0 .
\end{aligned}
$$




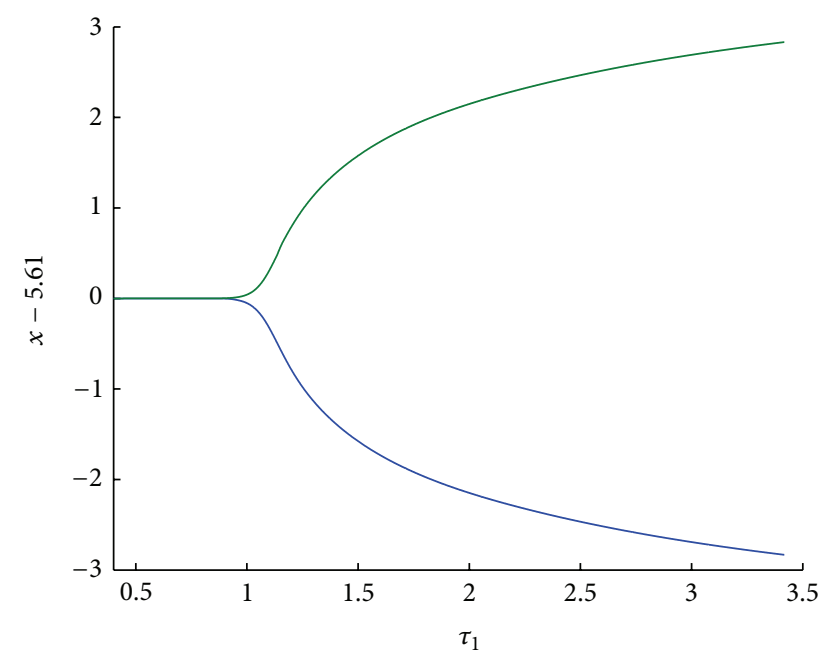

FIGURE 9: Bifurcation diagram with respect to the time delay $\tau_{1}$ for system (57) with $\tau_{2}=0$.
It is easy to see that if the condition (H2) holds, then all roots of (36) have a negative real part. Then the equilibrium point $E\left(x^{*}, y^{*}\right)$ is locally asymptotically stable when the conditions (H1) and (H2) are satisfied.

For $\theta>0$, let $i \theta$ be a root of (35). Then it follows that

$$
\begin{aligned}
& \left(p_{1} p_{4}-\theta^{2}+p_{3} p_{6}\right) \cos \theta \tau_{2}+\left(p_{3} p_{6}-p_{1} p_{4}+\theta^{2}\right) \sin \theta \tau \\
& \quad=p_{2} p_{5}-p_{3} p_{4}-p_{1} p_{6}, \\
& \left(p_{1}+p_{4}\right) \theta \cos \theta \tau+\left(p_{3} p_{6}-p_{1} p_{4}+\theta^{2}\right) \sin \theta \tau \\
& =-\left(p_{3}+p_{6}\right) \theta
\end{aligned}
$$

which is equivalent to

$$
\begin{aligned}
\sin \theta \tau & =\frac{\left(p_{2} p_{5}-p_{3} p_{4}-p_{1} p_{6}\right)\left(p_{1}+p_{4}\right) \theta+\left(p_{3}+p_{6}\right) \theta\left(p_{1} p_{4}-\theta^{2}+p_{3} p_{6}\right)}{\left[\left(p_{1}+p_{4}\right) \theta\right]^{2}-\left(p_{3} p_{6}\right)^{2}+\left(p_{1} p_{4}-\theta^{2}\right)^{2}}, \\
\cos \theta \tau & =\frac{\left(p_{3} p_{4}+p_{1} p_{6}-p_{2} p_{5}\right)\left(p_{3} p_{6}-p_{1} p_{4}+\theta^{2}\right)-\left(p_{3}+p_{6}\right) \theta\left(p_{1}+p_{4}\right) \theta}{\left[\left(p_{1}+p_{4}\right) \theta\right]^{2}-\left(p-3 p_{6}\right)^{2}+\left(p_{1} p_{4}-\theta^{2}\right)^{2}} .
\end{aligned}
$$

It follows from $\sin ^{2} \theta \tau+\cos ^{2} \theta \tau=1$ that

$$
\begin{aligned}
& {\left[\left(p_{2} p_{5}-p_{3} p_{4}-p_{1} p_{6}\right)\left(p_{1}+p_{4}\right) \theta\right.} \\
& \left.+\left(p_{3}+p_{6}\right) \theta\left(p_{1} p_{4}-\theta^{2}+p_{3} p_{6}\right)\right]^{2} \\
& +\left[\left(p_{3} p_{4}+p_{1} p_{6}-p_{2} p_{5}\right)\left(p_{3} p_{6}-p_{1} p_{4}+\theta^{2}\right)\right. \\
& \left.\quad-\left(p_{3}+p_{6}\right) \theta\left(p_{1}+p_{4}\right) \theta\right]^{2} \\
& =\left\{\left[\left(p_{1}+p_{4}\right) \theta\right]^{2}-\left(p_{3} p_{6}\right)^{2}+\left(p_{1} p_{4}-\theta^{2}\right)^{2}\right\}^{2} .
\end{aligned}
$$

Then we have

$$
\theta^{8}+u_{3} \theta^{6}+u_{2} \theta^{4}+u_{1} \theta^{2}+u_{0}=0
$$

where

$$
\begin{aligned}
u_{0}= & {\left[\left(p_{1} p_{4}\right)^{2}-\left(p_{3} p_{6}\right)^{2}\right]^{2} } \\
& -\left[\left(p_{2} p_{5}-p_{3} p_{4}-p_{1} p_{6}\right)\left(p_{3} p_{6}-p_{1} p_{4}\right)\right]^{2}, \\
u_{1}= & 2\left[\left(p_{1} p_{4}\right)^{2}-\left(p_{3} p_{6}\right)^{2}\right]\left[\left(p_{1}+p_{2}\right)^{2}-2 p_{1} p_{4}\right]^{2} \\
& -\left[\left(p_{2} p_{5}-p_{3} p_{4}-p_{1} p_{6}\right)\left(p_{1}+p_{4}\right)\right.
\end{aligned}
$$

$$
\begin{gathered}
\left.+\left(p_{3}+p_{6}\right)\left(p_{1} p_{4}+p_{3} p_{6}\right)\right] \\
-2\left(p_{2} p_{5}-p_{3} p_{4}-p_{1} p_{6}\right)\left(p_{1}+p_{4}\right) \\
\times\left[\left(p_{2} p_{5}-p_{3} p_{4}-p_{1} p_{6}\right)\right. \\
\left.\quad+\left(p_{3}+p_{6}\right)\left(p_{1}+p_{4}\right)\right] \\
u_{2}=2\left[\left(p_{1} p_{4}\right)^{2}-\left(p_{3} p_{6}\right)^{2}\right]+\left[\left(p_{1}+p_{2}\right)^{2}-2 p_{1} p_{4}\right]^{2} \\
+2\left[\left(p_{2} p_{5}-p_{3} p_{4}-p_{1} p_{6}\right)\left(p_{1}+p_{4}\right)\right. \\
\left.\quad+\left(p_{3}+p_{6}\right)\left(p_{1} p_{4}+p_{3} p_{6}\right)\right]\left(p_{3}+p_{6}\right) \\
-\left[\left(p-2 p_{5}-p_{3} p_{4}-p_{1} p_{6}\right)+\left(p_{3}+p_{6}\right)\left(p_{1}+p_{4}\right)\right]^{2}
\end{gathered}
$$$$
u_{3}=2\left[\left(p_{1}+p_{2}\right)^{2}-2 p_{1} p_{4}\right]-\left(p_{3}+p_{6}\right)^{2} \text {. }
$$

Let $z=\theta^{2}$. Then (41) becomes

$$
z^{4}+u_{3} z^{3}+u_{2} z^{2}+u_{1} z+u_{0}=0 .
$$

Denote

$$
h(z)=z^{4}+u_{3} z^{3}+u_{2} z^{2}+u_{1} z+u_{0} .
$$

Then

$$
h^{\prime}(z)=4 z^{3}+3 u_{3} z^{2}+2 u_{2} z+u_{1} .
$$




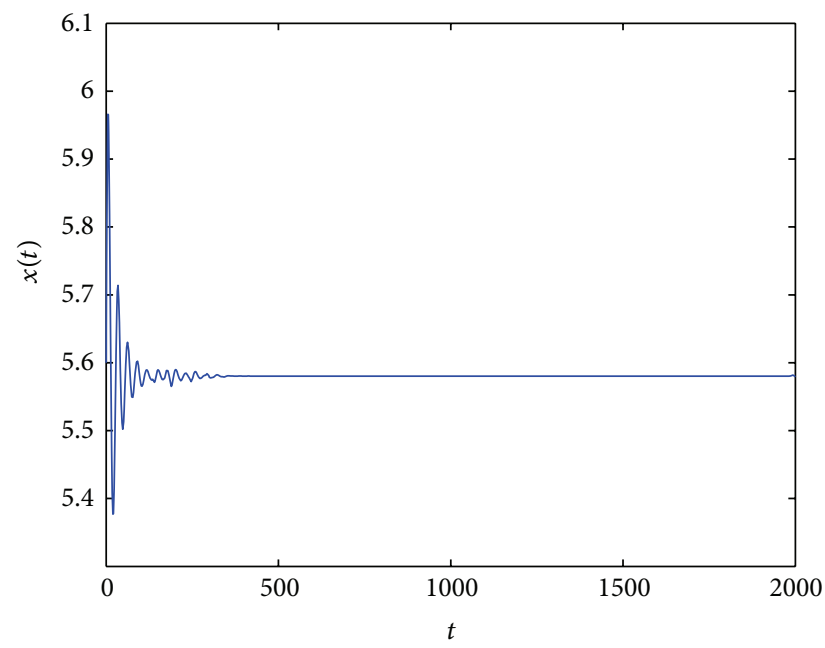

(a)

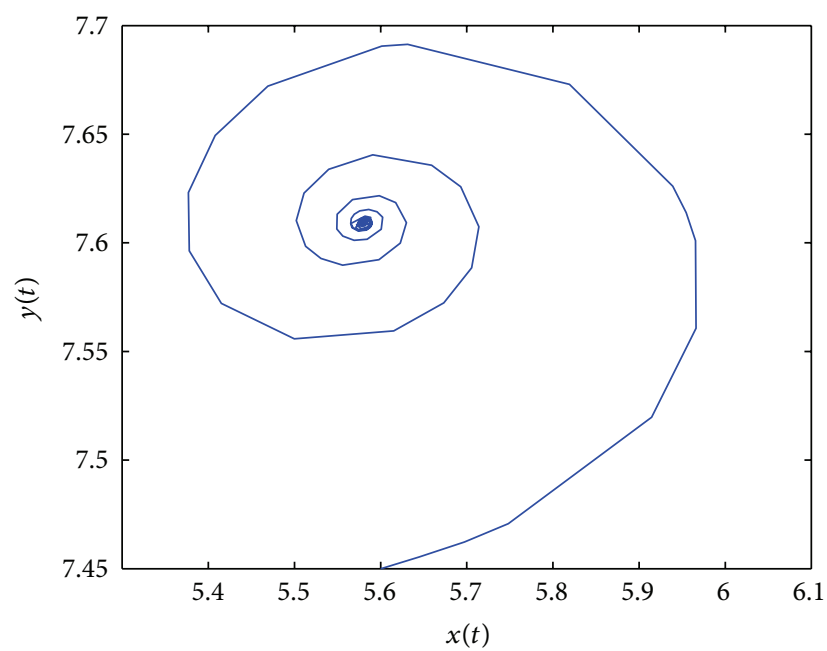

(c)

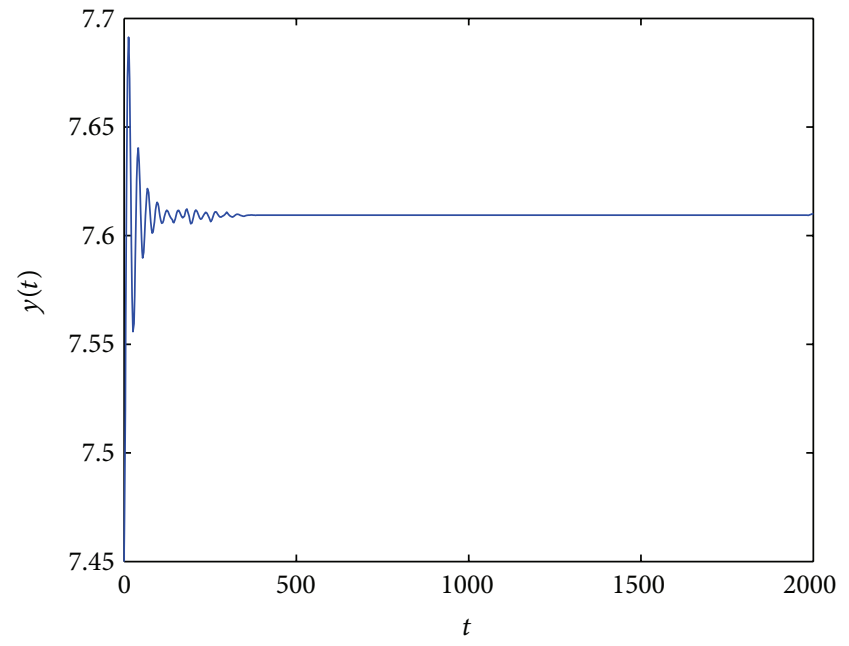

(b)

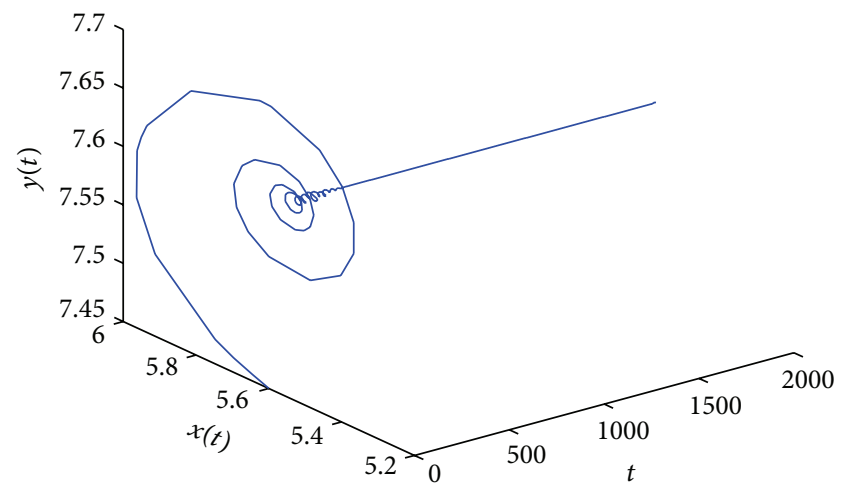

(d)

FIGURE 10: Trajectory portrait and phase portrait of system (57) with $\tau_{1}=0.5, \tau_{2}=0.5<\tau_{2_{0}} \approx 0.7723$. The positive equilibrium $E(5.61,7.02)$ is asymptotically stable. The initial value is $(5.6,7.45)$.

Set

$$
4 z^{3}+3 u_{3} z^{2}+2 u_{2} z+u_{1}=0
$$

Let $y=z+u_{3} / 4$. Then (46) becomes

$$
y^{3}+p_{1} y+q_{1}=0
$$

where

$$
p_{1}=\frac{u_{2}}{2}-\frac{3}{16} u_{3}^{2}, \quad q_{1}=\frac{u_{3}^{3}}{32}-\frac{u_{3} u_{2}}{8}+\frac{u_{1}}{4} .
$$

Define

$$
\begin{gathered}
D=\left(\frac{q_{1}}{2}\right)^{2}+\left(\frac{p_{1}}{3}\right)^{3}, \quad \sigma=\frac{-1+\sqrt{3}}{2}, \\
y_{1}=\sqrt[3]{-\frac{q_{1}}{2}+\sqrt{D}}+\sqrt[3]{-\frac{q_{1}}{2}-\sqrt{D}} \\
y_{2}=\sqrt[3]{-\frac{q_{1}}{2}+\sqrt{D} \sigma}+\sqrt[3]{-\frac{q_{1}}{2}-\sqrt{D} \sigma^{2}} \\
y_{3}=\sqrt[3]{-\frac{q_{1}}{2}+\sqrt{D} \sigma^{2}}+\sqrt[3]{-\frac{q_{1}}{2}-\sqrt{D} \sigma} \\
z_{i}=y_{i}-\frac{p_{1}}{4}, \quad i=1,2,3 .
\end{gathered}
$$




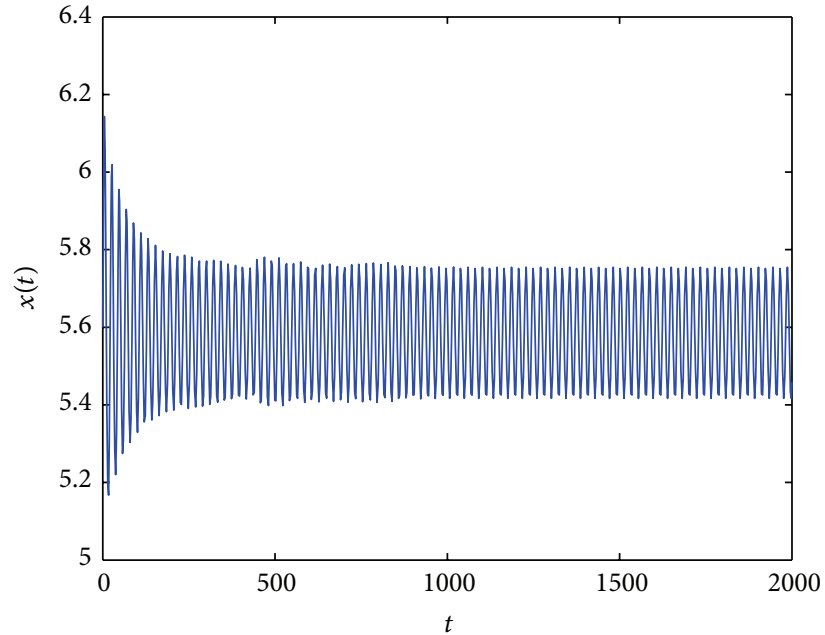

(a)

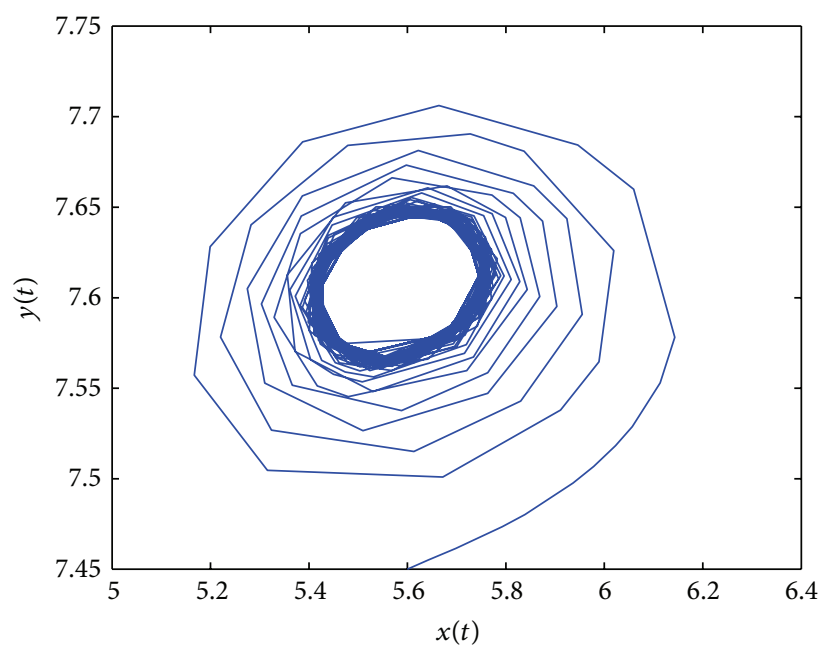

(c)

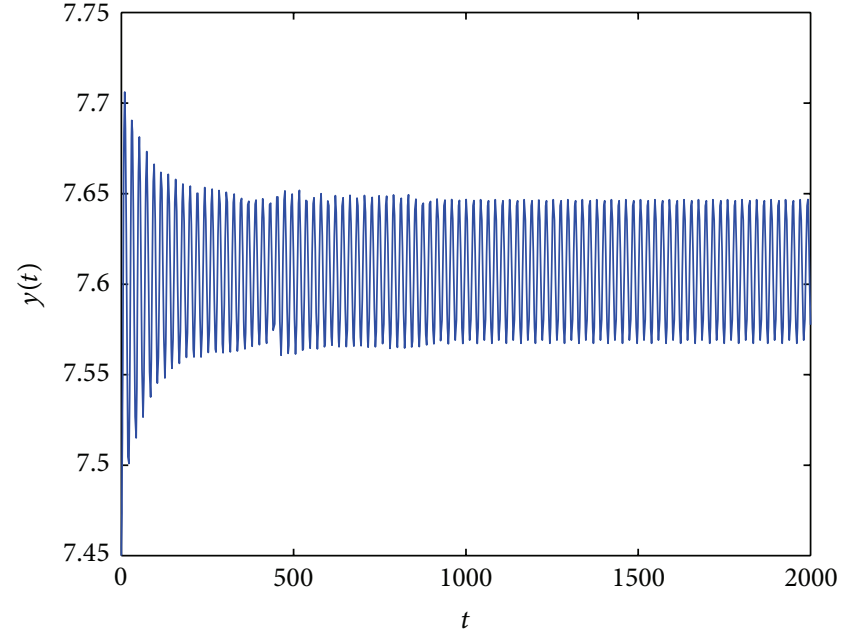

(b)

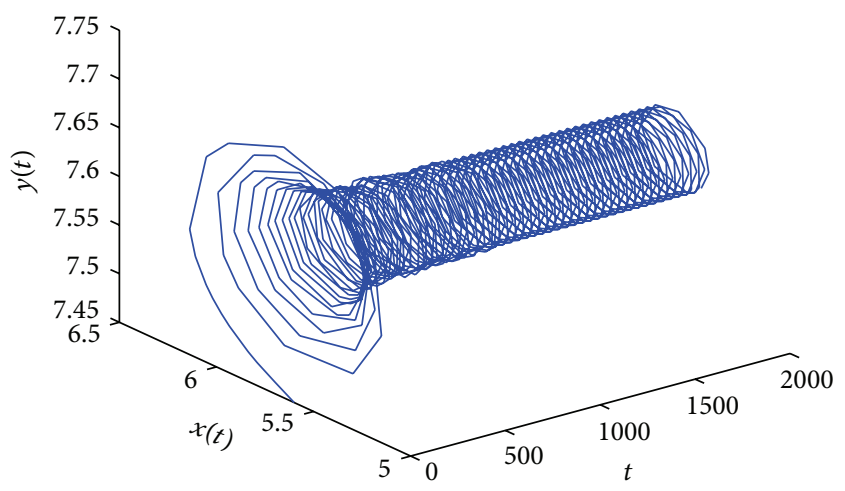

(d)

FIGURE 11: Trajectory portrait and phase portrait of system (57) with $\tau_{1}=0.5, \tau_{2}=1.6>\tau_{2_{0}} \approx 0.7723$. Hopf bifurcation occurs from the positive equilibrium $E(5.61,7.02)$. The initial value is $(5.6,7.45)$.

From $[32,33]$, we have the following result.

Lemma 8. If $u_{0}<0$, then (43) has at least one positive root.

Lemma 9. Assume that $u_{0} \geq 0$. Then one has the following:

(i) if $D \geq 0$, then (43) has positive roots if and only if $z_{1}>0$ and $h^{\prime}\left(z_{1}\right)<0$;

(ii) if $D<0$, then (43) has positive roots if and only if there exists at least one $z^{*} \in\left\{z_{1}, z_{2}, z_{3}\right\}$ such that $z^{*}>0$ and $h^{\prime}\left(z^{*}\right) \leq 0$.
Without loss of generality, we assume that (43) has four positive roots, defined by $z_{1}, z_{2}, z_{3}, z_{4}$, respectively. Then (41) has four positive roots

$$
\begin{array}{ll}
\theta_{1}=\sqrt{z_{1}}, & \theta_{2}=\sqrt{z_{2}}, \\
\theta_{3}=\sqrt{z_{3}}, & \theta_{4}=\sqrt{z_{4}} .
\end{array}
$$

By (39), if we denote

$$
\tau_{k}^{(j)}=\frac{1}{\theta_{k}}\left\{\arccos \left[\frac{\left(p_{3} p_{4}+p_{1} p_{6}-p_{2} p_{5}\right)\left(p_{3} p_{6}-p_{1} p_{4}+\theta^{2}\right)-\left(p_{3}+p_{6}\right) \theta\left(p_{1}+p_{4}\right) \theta}{\left[\left(p_{1}+p_{4}\right) \theta\right]^{2}-\left(p-3 p_{6}\right)^{2}+\left(p_{1} p_{4}-\theta^{2}\right)^{2}}\right]+2 j \pi\right\},
$$




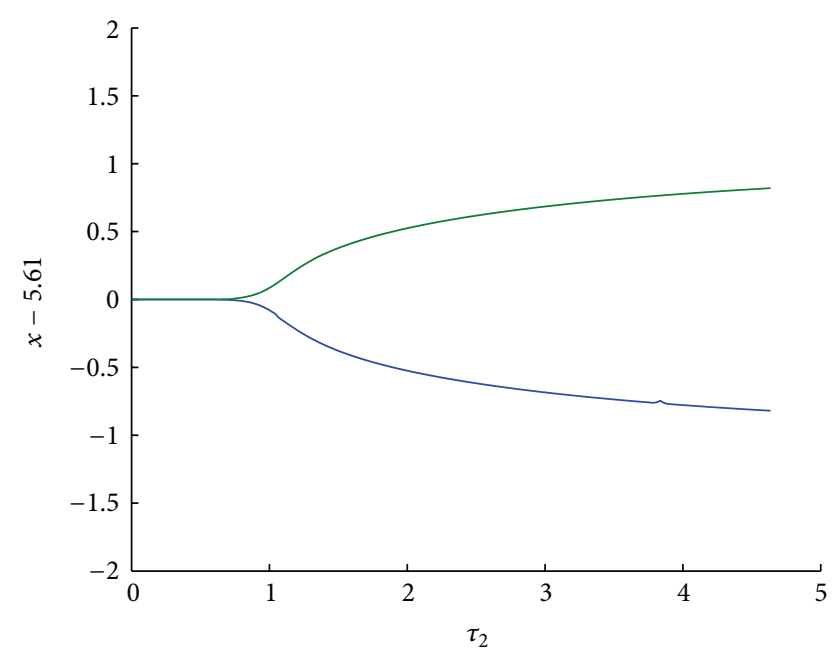

FIGURE 12: Bifurcation diagram with respect to the time delay $\tau_{2}$ for system (57) with $\tau_{1}=0.5$.

where $k=1,2,3,4 ; j=0,1, \ldots$, then $\pm i \theta_{k}$ are a pair of purely imaginary roots of (35) with $\tau_{k}^{(j)}$. Define

$$
\tau_{0}=\tau_{k_{0}}^{(0)}=\min _{k \in\{1,2,3,4\}}\left\{\tau_{k}^{(0)}\right\}, \quad \theta_{0}=\theta_{k_{0}}
$$

Based on above analysis, we have the following result.

Lemma 10. For $\tau_{1}=\tau_{2}=\tau$, if (H1) and (H2) hold, then all roots of (1) have a negative real part when $\tau \in\left[0, \tau_{0}\right)$, and (1) admits a pair of purely imaginary roots $\pm \theta_{k} i$ when $\tau=\tau_{k}^{(j)}(k=$ $1,2,3,4, j=0,1,2, \ldots)$.

Let $\lambda(\tau)=\alpha(\tau)+i \theta(\tau)$ be a root of (35) near $\tau=\tau_{k}^{(j)}$, and let $\alpha\left(\tau_{k}^{(j)}\right)=0$ and $\theta\left(\tau_{k}^{(j)}\right)=\theta_{k}$. Due to functional differential equation theory, for every $\tau_{k}^{(j)}, k=1,2,3,4, j=0,1,2, \ldots$, there exists $\varepsilon>0$ such that $\lambda(\tau)$ is continuously differentiable in $\tau$ for $\left|\tau-\tau_{k}^{(j)}\right|<\varepsilon$. Substituting $\lambda(\tau)$ into the left-hand side of (35) and taking derivative with respect to $\tau$, we have

$$
\begin{aligned}
{\left[\frac{d \lambda}{d \tau}\right]^{-1} } \\
=-\frac{\left[2 \lambda-\left(p_{1}+p_{4}\right)\right] \lambda e^{\lambda \tau}-\left(p_{2}+p_{6}\right)+p_{3} p_{6} e^{-\lambda \tau}}{\lambda\left[\lambda^{2}-\left(p_{1}+p_{4}\right) \lambda+p_{1} p_{4}\right] e^{\lambda \tau}+p_{3} p_{6} \lambda^{2} e^{-\lambda \tau}} \\
\quad-\frac{\tau}{\lambda} .
\end{aligned}
$$

We can easily obtain

$$
\left[\frac{d(\operatorname{Re} \lambda(\tau))}{d \tau}\right]_{\tau=\tau_{k}^{(j)}}^{-1}
$$

$$
\begin{aligned}
& =-\operatorname{Re}\left\{\frac{\left[2 \lambda-\left(p_{1}+p_{4}\right)\right] \lambda e^{\lambda \tau}-\left(p_{2}+p_{6}\right)+p_{3} p_{6} e^{-\lambda \tau}}{\lambda\left[\lambda^{2}-\left(p_{1}+p_{4}\right) \lambda+p_{1} p_{4}\right] e^{\lambda \tau}+p_{3} p_{6} \lambda^{2} e^{-\lambda \tau}}\right\}_{\tau=\tau_{k}^{(j)}} \\
& =-\operatorname{Re}\left\{\frac{A_{1}-A_{2} i}{B_{1}+B_{2} i}\right\}=-\frac{A_{1} B_{1}-A_{2} B_{2}}{B_{1}^{2}+B_{2}^{2}}
\end{aligned}
$$

where

$$
\begin{aligned}
A_{1}= & \left(p_{3} p_{6}-2 \theta_{k}^{2}\right) \cos \theta_{k} \tau_{k}^{(j)} \\
& +\left(p_{1}+p_{4}\right) \theta_{k} \sin \theta_{k} \tau_{k}^{(j)}-\left(p_{2}+p_{6}\right), \\
A_{2}= & \left(p_{1}+p_{4}\right) \theta_{k} \tau_{k}^{(j)} \cos \theta_{k} \tau_{k}^{(j)}+\left(2 \theta_{k}^{2}+p_{3} p_{6}\right) \sin \theta_{k} \tau_{k}^{(j)}, \\
B_{1}= & \left(p_{1}+p_{4}\right) \theta_{k}^{2} \cos \theta_{k} \tau_{k}^{(j)} \\
& -\left(p_{1} p_{4}-\theta_{k}^{2}\right) \theta_{k} \sin \theta_{k} \tau_{k}^{(j)}-p_{3} p_{6} \theta_{k}^{2}, \\
B_{2}= & \left(p_{1}+p_{4}\right) \theta_{k}^{2} \sin \theta_{k} \tau_{k}^{(j)}+\left(p_{1} p_{4}-\theta_{k}^{2}\right) \theta_{k} \cos \theta_{k} \tau_{k}^{(j)} \\
& +p_{3} p_{6} \theta_{k}^{2} \sin \theta_{k} \tau_{k}^{(j)} .
\end{aligned}
$$

Now we assume that

(H6)

$$
A_{1} B_{1} \neq A_{2} B_{2} \text {. }
$$

In view of the above analysis and the results of Kuang [33] and Hale [31], we have the following.

Theorem 11. For $\tau=0$, if (H1) and (H2) hold, then the positive equilibrium $E\left(x^{*}, y^{*}\right)$ of system (1) is asymptotically stable for $\tau \in\left[0, \tau_{0}\right)$. In addition to the conditions ( $\mathrm{H1}$ ) and (H2), one further assumes that (H6) holds. Then system (1) undergoes a Hopf bifurcation at the positive equilibrium $E\left(x^{*}, y^{*}\right)$ when $\tau=\tau_{k}^{(j)}, k=1,2,3,4, j=0,1,2, \ldots$.

\section{Computer Simulations}

In this section, we present some numerical results of system (1) to verify the analytical predictions obtained in the previous section. Let us consider the following system:

$$
\begin{aligned}
& \dot{x}(t)=2 x\left(t-\tau_{1}\right)-0.12 x^{2}-\frac{2 x y}{0.77 y+x}, \\
& \dot{y}(t)=\frac{0.205 x\left(t-\tau_{2}\right) y\left(t-\tau_{2}\right)}{0.77 y\left(t-\tau_{2}\right)+x\left(t-\tau_{2}\right)}-0.1 y,
\end{aligned}
$$

which has a positive equilibrium $E(5.61,7.02)$. We can easily obtain that (H1)-(H6) hold true. When $\tau_{1}=0$, applying MATLAB 7.0, we can get $\omega_{0} \approx 0.5524, \tau_{2_{0}} \approx 2.3345$. The positive equilibrium $E(5.61,7.02)$ is asymptotically stable for $\tau_{2}<\tau_{2_{0}} \approx 2.3345$ and unstable for $\tau_{2}>\tau_{2_{0}} \approx 2.3345$ which is shown in Figure 1. When $\tau_{2}=\tau_{2_{0}} \approx 2.3345$, (57) undergoes a Hopf bifurcation around the positive equilibrium 


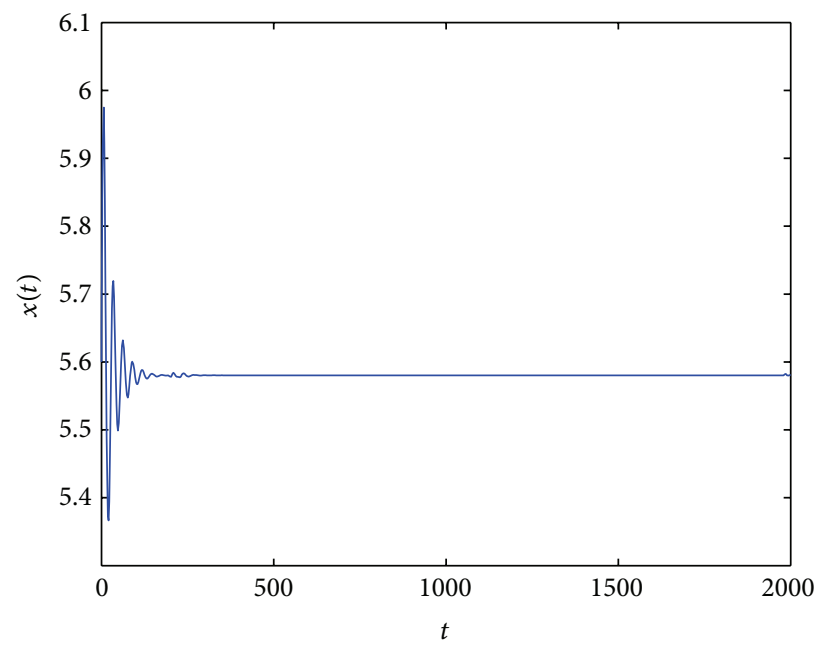

(a)

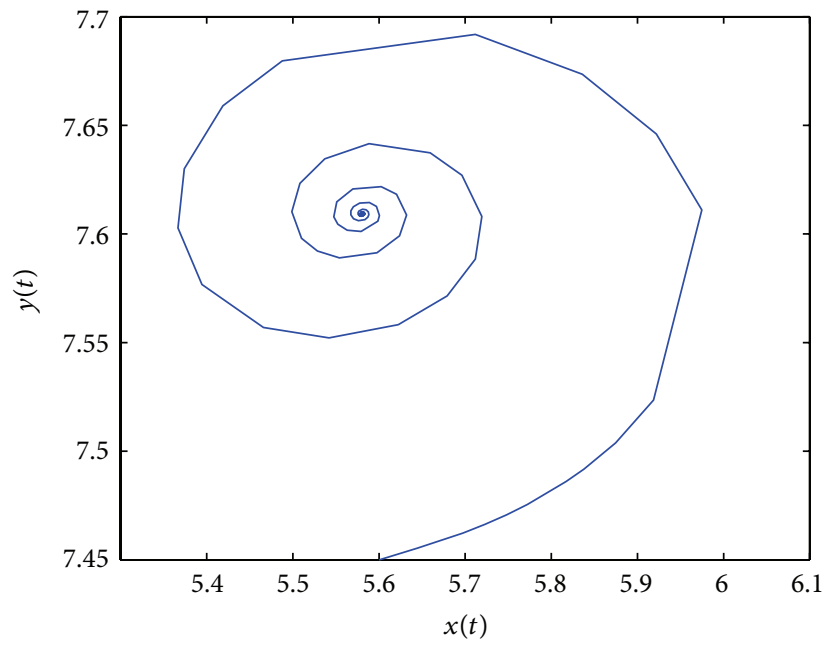

(c)

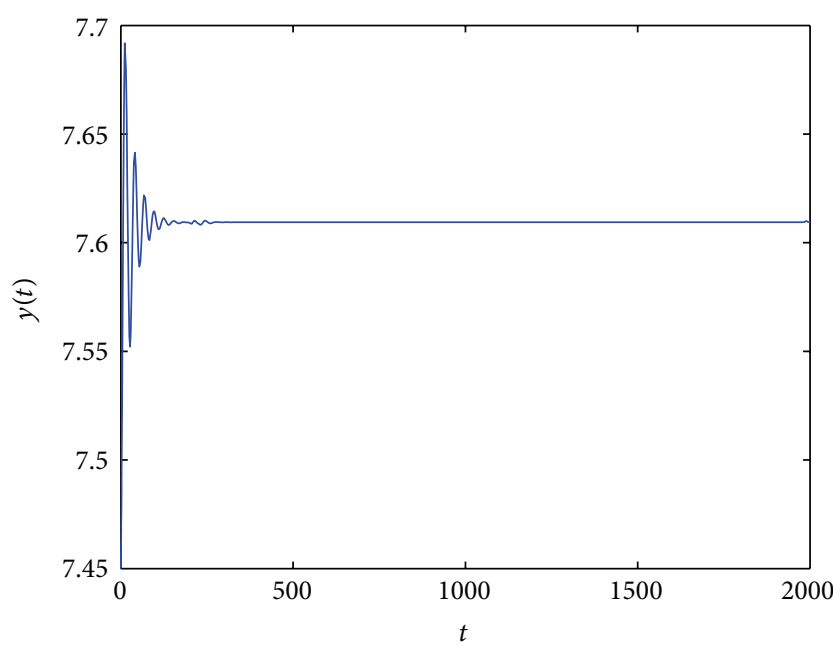

(b)

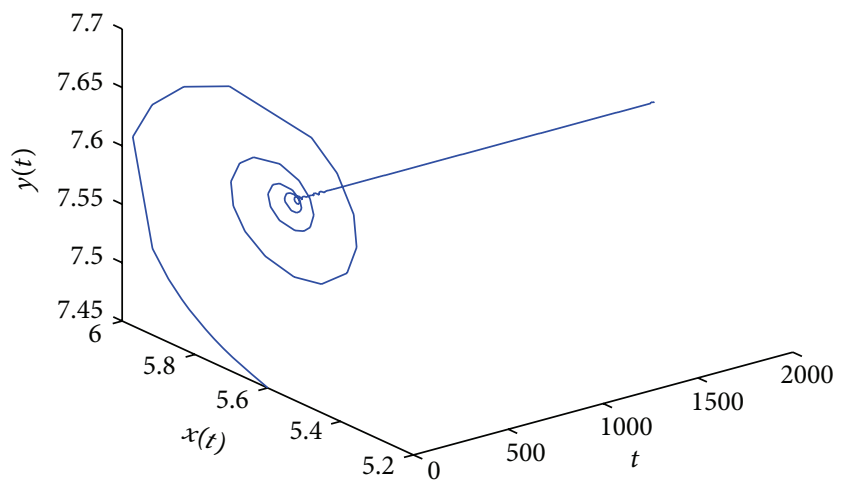

(d)

FIGURE 13: Trajectory portrait and phase portrait of system (57) with $\tau_{1}=0 \tau_{2}=\tau=1.0<\tau_{0} \approx 1.2032$. The positive equilibrium $E(5.61,7.02)$ is asymptotically stable. The initial value is $(5.6,7.45)$.

$E(5.61,7.02)$. That is, a small amplitude periodic solution occurs near $E(5.61,7.02)$ when $\tau_{1}=0$ and $\tau_{2}$ is close to $\tau_{2_{0}}=$ 2.3345 which is shown in Figure 2. The bifurcation diagram of the case is shown in Figure 3.

Let $\tau_{2}=0.25 \in(0,1.8)$, and choose $\tau_{1}$ as a parameter. We have $\tau_{1_{0}} \approx 0.8013$. Then the positive equilibrium is asymptotically stable when $\tau_{1} \in\left[0, \tau_{1_{0}}\right)$. The Hopf bifurcation value of (57) is $\tau_{1_{0}} \approx 0.8013$ (see Figures 4 and 5) The bifurcation diagram of the case is shown in Figure 6.

When $\tau_{2}=0$, using MATLAB 7.0, we obtain $\eta_{0} \approx 0.9056$, $\tau_{1_{0}} \approx 0.9122$. The positive equilibrium $E(5.61,7.02)$ is asymptotically stable for $\tau_{1}<\tau_{1_{0}} \approx 0.9122$ and unstable for $\tau_{1}>$ $\tau_{1_{0}} \approx 0.9122$ which is shown in Figure 7. When $\tau_{1}=\tau_{1_{0}} \approx$ 0.9122 , (57) undergoes a Hopf bifurcation at the positive equilibrium $E_{0}(5.61,7.02)$. That is, a small amplitude periodic solution occurs around $E(5.61,7.02)$ when $\tau_{2}=0$ and $\tau_{1}$ is close to $\tau_{1_{0}}=0.9122$ which is illustrated in Figure 8 . The bifurcation diagram of the case is shown in Figure 9.

Let $\tau_{1}=0.5 \in(0,0.9122)$, and choose $\tau_{2}$ as a parameter. We have $\tau_{2} \approx 0.7723$. Then the positive equilibrium is asymptotically stable when $\tau_{2} \in\left[0, \tau_{2_{0}}\right)$. The Hopf bifurcation value of (57) is $\tau_{2_{0}} \approx 0.7723$ (see Figures 10 and 11). The bifurcation diagram of the case is shown in Figure 12.

When $\tau_{1}=\tau_{2}=\tau$, using MATLAB 7.0, we obtain $\theta_{0} \approx$ $0.8725, \tau_{0} \approx 1.2032$. The positive equilibrium $E(5.61,7.02)$ is asymptotically stable for $\tau<\tau_{0} \approx 1.2032$ and unstable for $\tau>\tau_{0} \approx 1.2032$ which is shown in Figure 13. When $\tau=\tau_{0} \approx 1.2032$, (57) undergoes a Hopf bifurcation at the positive equilibrium $E_{0}(5.61,7.02)$. That is, a small amplitude periodic solution occurs around $E(5.61,7.02)$ when $\tau$ is close to $\tau_{0} \approx 1.2032$ which is illustrated in Figure 14 . The bifurcation diagram of the case is shown in Figure 15. 


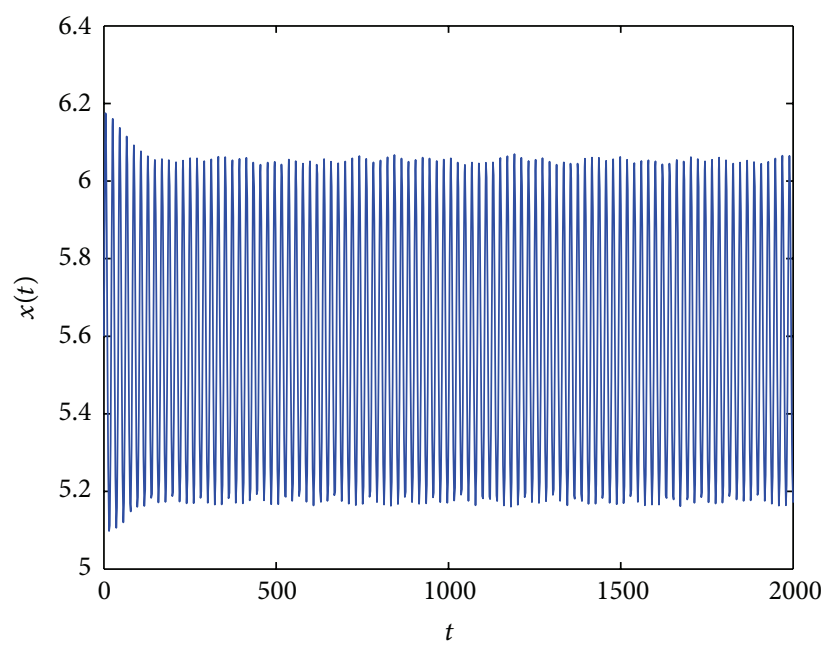

(a)

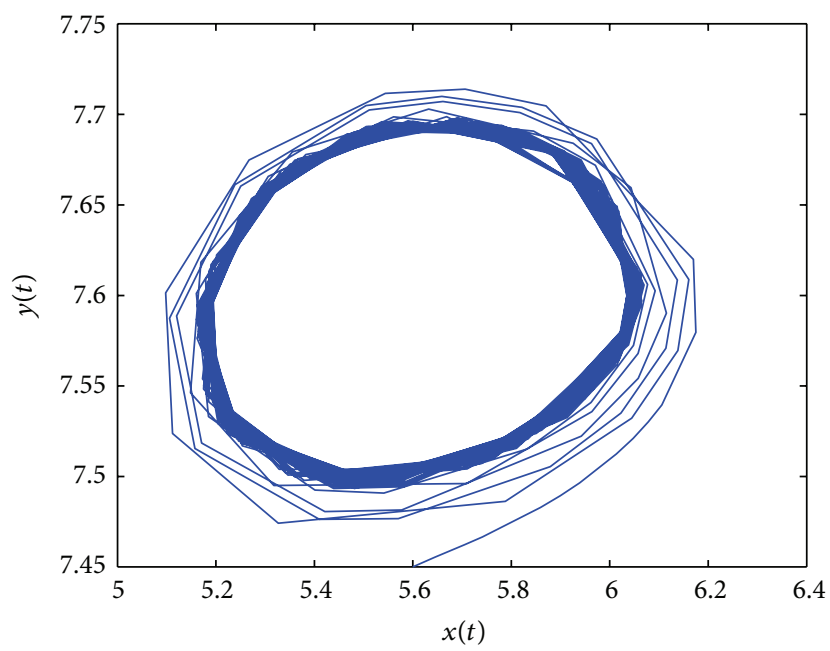

(c)

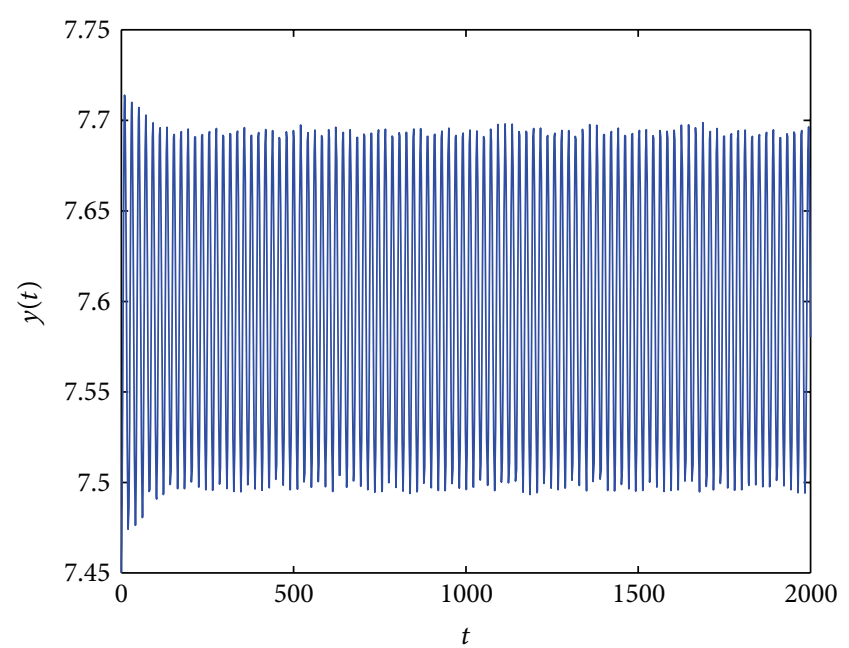

(b)

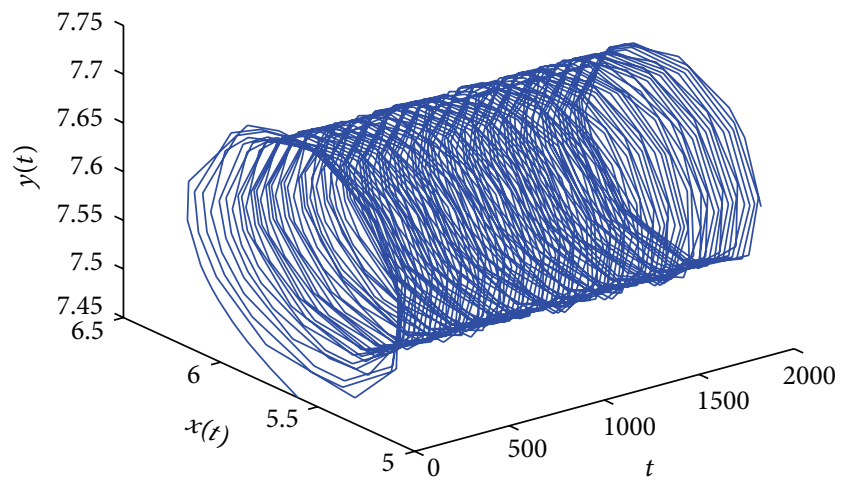

(d)

FIGURE 14: Trajectory portrait and phase portrait of system (57) with $\tau_{1}=\tau_{2}=\tau=1.25>\tau_{0} \approx 1.2032$. Hopf bifurcation occurs from the positive equilibrium $E(5.61,7.02)$. The initial value is $(5.6,7.45)$.

\section{Conclusions}

In this paper, we have investigated local stability of the positive equilibrium $E\left(x^{*}, y^{*}\right)$ and local Hopf bifurcation of a ratio-dependent predator-prey model with two delays. It is shown that if some conditions hold true and $\tau_{2} \in\left[0, \tau_{2_{0}}\right)$, then the positive equilibrium $E\left(x^{*}, y^{*}\right)$ is asymptotically stable when $\tau_{1} \in\left(0, \tau_{1_{0}}\right)$. When the delay $\tau_{1}$ increases, the positive equilibrium $E\left(x^{*}, y^{*}\right)$ loses its stability and a sequence of Hopf bifurcations occur at the positive equilibrium $E\left(x^{*}, y^{*}\right)$. That is, a family of periodic orbits bifurcates from the the positive equilibrium $E\left(x^{*}, y^{*}\right)$. We also showed that if a certain condition is satisfied and $\tau_{1} \in\left[0, \tau_{1_{0}}\right)$, then the positive equilibrium $E\left(x^{*}, y^{*}\right)$ is asymptotically stable when $\tau_{2} \in\left(0, \tau_{2_{0}}\right)$, when the delay $\tau_{2}$ increases, the positive equilibrium $E\left(x^{*}, y^{*}\right)$ loses its stability and a sequence of Hopf bifurcations occur at the positive equilibrium $E\left(x^{*}, y^{*}\right)$.
In case $\tau_{1}=\tau_{2}=\tau$, we have shown that if some conditions are satisfied, and $\tau \in\left[0, \tau_{0}\right)$, then the positive equilibrium $E\left(x^{*}, y^{*}\right)$ is asymptotically stable when $\tau \in\left(0, \tau_{0}\right)$. When the delay $\tau$ increases, the positive equilibrium $E\left(x^{*}, y^{*}\right)$ loses its stability and a sequence of Hopf bifurcations occur at the positive equilibrium $E\left(x^{*}, y^{*}\right)$ which means a family of periodic orbits bifurcates from the the positive equilibrium $E\left(x^{*}, y^{*}\right)$. Some numerical simulations verifying our theoretical results are carried out. In addition, we must point out that although Ko and Ryu [6] have also investigated the the existence of Hopf bifurcation for system (1) with respect to positive equilibrium $E\left(x^{*}, y^{*}\right)$, it is assumed that $\tau_{1}+\tau_{2}$ in a certain range and choose the delay $\tau_{2}$ as bifurcation parameter to consider the Hopf bifurcation nature. But what effect different time delays have on the dynamics of system (1)? Ko and Ryu [6] did not deal with this issue. It is important for us to consider what effect the two different time delays has 


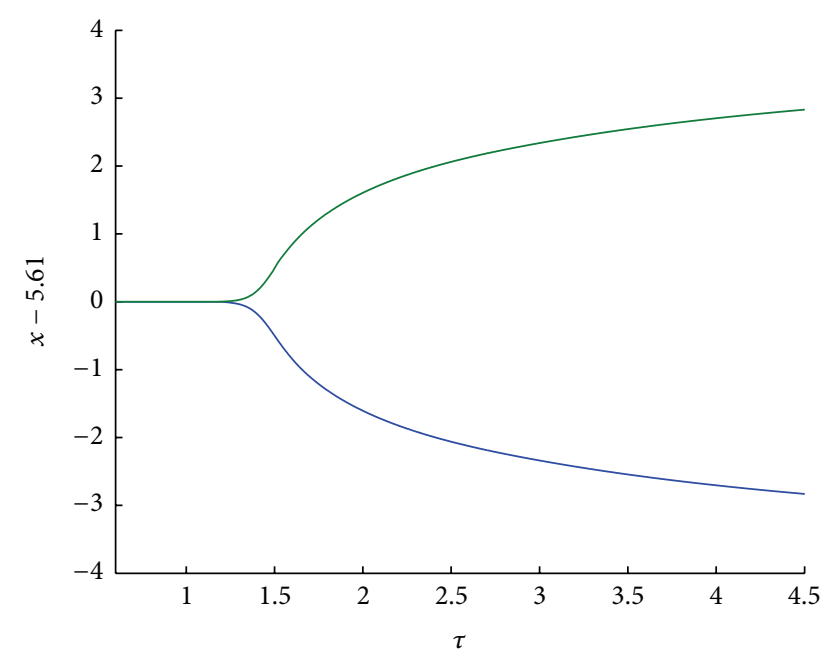

Figure 15: Bifurcation diagram with respect to the time delay $\tau$ for system (57) with $\tau_{1}=\tau_{2}=\tau$.

on the dynamical behavior of system (1). Thus we think that our work generalizes the known results of Ko and Ryu [6]. In addition, we can study the Hopf bifurcation nature of system (1) by regarding the delay $\tau_{2}$ as bifurcation parameter. We will further focus on the topic elsewhere in the near future.

\section{Acknowledgments}

This work is supported by the National Natural Science Foundation of China (no. 11261010 and no. 11101126), the Soft Science and Technology Program of Guizhou Province (no. 2011LKC2030), Natural Science and Technology Foundation of Guizhou Province (J[2012]2100), Governor Foundation of Guizhou Province ([2012]53) and Doctoral Foundation of Guizhou University of Finance and Economics (2010).

\section{References}

[1] R. Bhattacharyya and B. Mukhopadhyay, "On an eco-epidemiological model with prey harvesting and predator switching: local and global perspectives," Nonlinear Analysis: Real World Applications, vol. 11, no. 5, pp. 3824-3833, 2010.

[2] T. K. Kar and A. Ghorai, "Dynamic behaviour of a delayed predator-prey model with harvesting," Applied Mathematics and Computation, vol. 217, no. 22, pp. 9085-9104, 2011.

[3] K. Chakraborty, M. Chakraborty, and T. K. Kar, "Bifurcation and control of a bioeconomic model of a prey-predator system with a time delay," Nonlinear Analysis: Hybrid Systems, vol. 5, no. 4, pp. 613-625, 2011.

[4] R. Bhattacharyya and B. Mukhopadhyay, "Spatial dynamics of nonlinear prey-predator models with prey migration and predator switching," Ecological Complexity, vol. 3, no. 2, pp. 160 169, 2006.

[5] X. Chang and J. Wei, "Hopf bifurcation and optimal control in a diffusive predator-prey system with time delay and prey harvesting," Lithuanian Association of Nonlinear Analysts (LANA), vol. 17, no. 4, pp. 379-409, 2012.
[6] W. Ko and K. Ryu, "Coexistence states of a nonlinear LotkaVolterra type predator-prey model with cross-diffusion," Nonlinear Analysis: Theory, Methods \& Applications, vol. 71, no. 12, pp. e1109-e1115, 2009.

[7] S. Gao, L. Chen, and Z. Teng, "Hopf bifurcation and global stability for a delayed predator-prey system with stage structure for predator," Applied Mathematics and Computation, vol. 202, no. 2, pp. 721-729, 2008.

[8] T. K. Kar and U. K. Pahari, "Modelling and analysis of a preypredator system with stage-structure and harvesting," Nonlinear Analysis: Real World Applications, vol. 8, no. 2, pp. 601-609, 2007.

[9] Y. Kuang and Y. Takeuchi, "Predator-prey dynamics in models of prey dispersal in two-patch environments," Mathematical Biosciences, vol. 120, no. 1, pp. 77-98, 1994.

[10] K. Li and J. Wei, "Stability and Hopf bifurcation analysis of a prey-predator system with two delays," Chaos, Solitons \& Fractals, vol. 42, no. 5, pp. 2606-2613, 2009.

[11] R. M. May, "Time delay versus stability in population models with two and three trophic levels," Ecology, vol. 54, no. 2, pp. 315-325, 1973.

[12] Prajneshu and P. Holgate, "A prey-predator model with switching effect," Journal of Theoretical Biology, vol. 125, no. 1, pp. 6166, 1987.

[13] S. Ruan, "Absolute stability, conditional stability and bifurcation in Kolmogorov-type predator-prey systems with discrete delays," Quarterly of Applied Mathematics, vol. 59, no. 1, pp. 159173, 2001.

[14] Y. Song and J. Wei, "Local Hopf bifurcation and global periodic solutions in a delayed predator-prey system," Journal of Mathematical Analysis and Applications, vol. 301, no. 1, pp. 1-21, 2005.

[15] E. Teramoto, K. Kawasaki, and N. Shigesada, "Switching effect of predation on competitive prey species," Journal of Theoretical Biology, vol. 79, no. 3, pp. 303-315, 1979.

[16] R. Xu, M. A. J. Chaplain, and F. A. Davidson, "Periodic solutions for a delayed predator-prey model of prey dispersal in two-patch environments," Nonlinear Analysis: Real World Applications, vol. 5, no. 1, pp. 183-206, 2004.

[17] R. Xu and Z. Ma, "Stability and Hopf bifurcation in a ratiodependent predator-prey system with stage structure," Chaos, Solitons \& Fractals, vol. 38, no. 3, pp. 669-684, 2008.

[18] T. Zhao, Y. Kuang, and H. L. Smith, "Global existence of periodic solutions in a class of delayed Gause-type predator-prey systems," Nonlinear Analysis: Theory, Methods \& Applications, vol. 28, no. 8, pp. 1373-1394, 1997.

[19] X. Zhou, X. Shi, and X. Song, "Analysis of nonautonomous predator-prey model with nonlinear diffusion and time delay," Applied Mathematics and Computation, vol. 196, no. 1, pp. 129136, 2008.

[20] N. Bairagi and D. Jana, "On the stability and Hopf bifurcation of a delay-induced predator-prey system with habitat complexity," Applied Mathematical Modelling, vol. 35, no. 7, pp. 3255-3267, 2011.

[21] L. Zhang and C. Lu, "Periodic solutions for a semi-ratiodependent predator-prey system with Holling IV functional response," Journal of Applied Mathematics and Computing, vol. 32, no. 2, pp. 465-477, 2010.

[22] X. Tian and R. Xu, "Global dynamics of a predator-prey system with Holling type II functional response," Lithuanian Association of Nonlinear Analysts (LANA), vol. 16, no. 2, pp. 242253,2011 . 
[23] M. Xiao and J. Cao, "Hopf bifurcation and non-hyperbolic equilibrium in a ratio-dependent predator-prey model with linear harvesting rate: analysis and computation," Mathematical and Computer Modelling, vol. 50, no. 3-4, pp. 360-379, 2009.

[24] Y. Xia, J. Cao, and M. Lin, "Discrete-time analogues of predatorprey models with monotonic or nonmonotonic functional responses," Nonlinear Analysis: Real World Applications, vol. 8, no. 4, pp. 1079-1095, 2007.

[25] Z. Cheng, Y. Lin, and J. Cao, "Dynamical behaviors of a partialdependent predator-prey system," Chaos, Solitons and Fractals, vol. 28, no. 1, pp. 67-75, 2006.

[26] M. Xiao and J. Cao, "Genetic oscillation deduced from Hopf bifurcation in a genetic regulatory network with delays," Mathematical Biosciences, vol. 215, no. 1, pp. 55-63, 2008.

[27] W.-Y. Wang and L.-J. Pei, "Stability and Hopf bifurcation of a delayed ratio-dependent predator-prey system," Acta Mechanica Sinica, vol. 27, no. 2, pp. 285-296, 2011.

[28] S. Ruan and J. Wei, "On the zeros of transcendental functions with applications to stability of delay differential equations with two delays," Dynamics of Continuous, Discrete \& Impulsive Systems A, vol. 10, no. 6, pp. 863-874, 2003.

[29] J. Cao and M. Xiao, "Stability and Hopf bifurcation in a simplified BAM neural network with two time delays," IEEE Transactions on Neural Networks, vol. 18, no. 2, pp. 416-430, 2007.

[30] Z. Ge and J. Yan, "Hopf bifurcation of a predator-prey system with stage structure and harvesting," Nonlinear Analysis: Theory, Methods \& Applications, vol. 74, no. 2, pp. 652-660, 2011.

[31] J. Hale, Theory of Functional Differential Equations, vol. 3, Springer, Berlin, Germany, 2nd edition, 1977.

[32] H. Hu and L. Huang, "Stability and Hopf bifurcation analysis on a ring of four neurons with delays," Applied Mathematics and Computation, vol. 213, no. 2, pp. 587-599, 2009.

[33] Y. Kuang, Delay Differential Equations with Applications in Population Dynamics, vol. 191 of Mathematics in Science and Engineering, Academic Press; INC, Boston, Mass, USA, 1993. 


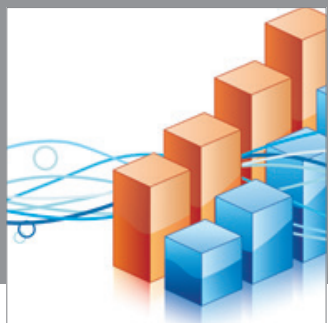

Advances in

Operations Research

mansans

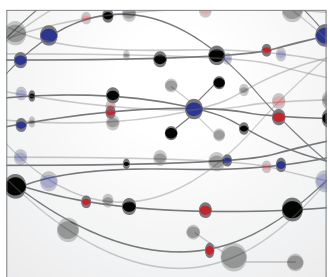

The Scientific World Journal
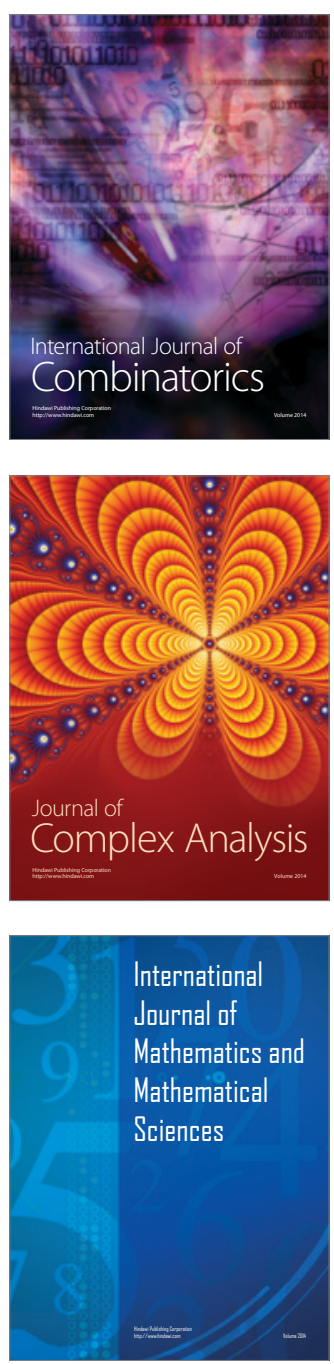
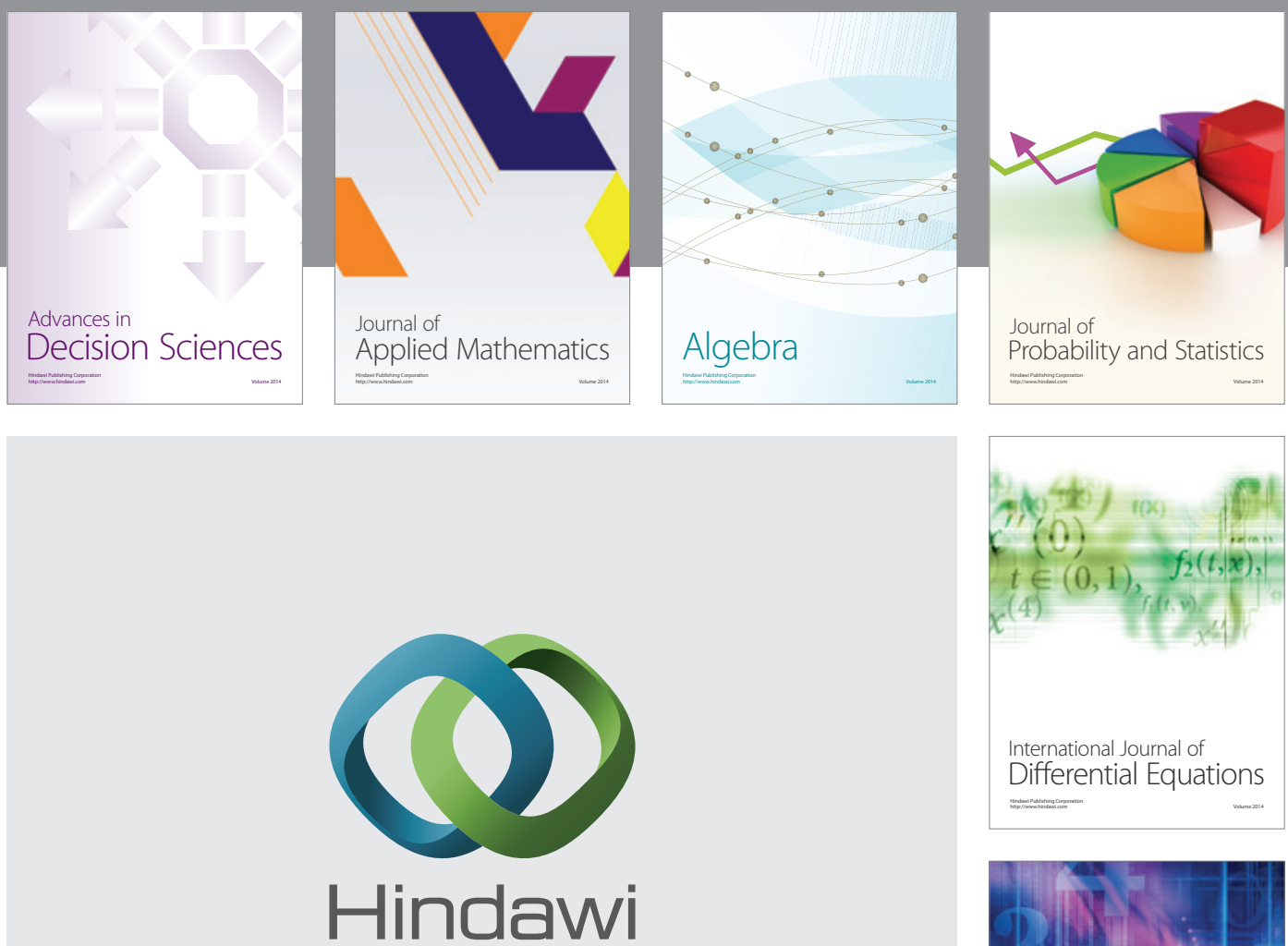

Submit your manuscripts at http://www.hindawi.com
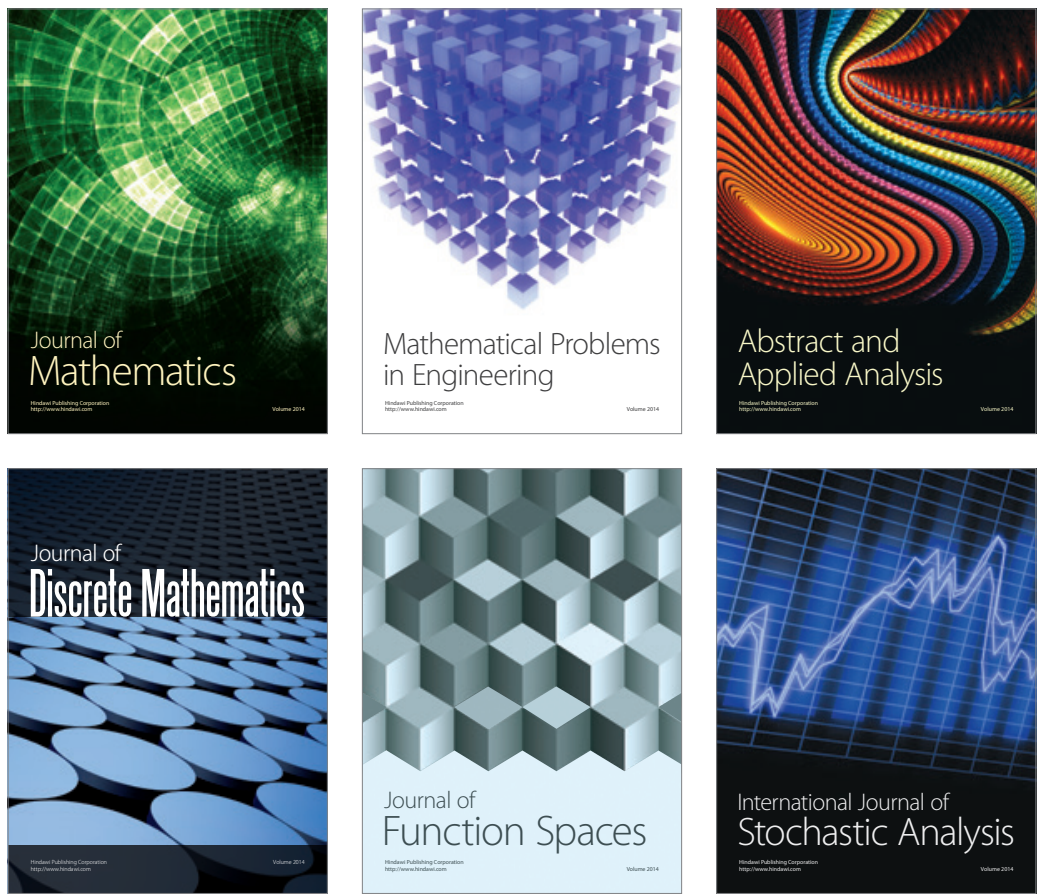

Journal of

Function Spaces

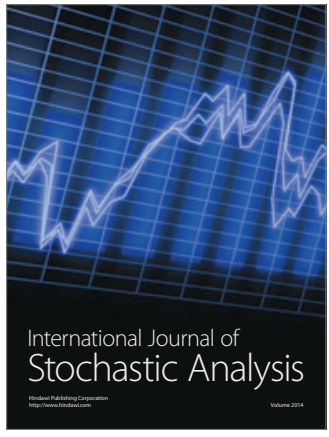

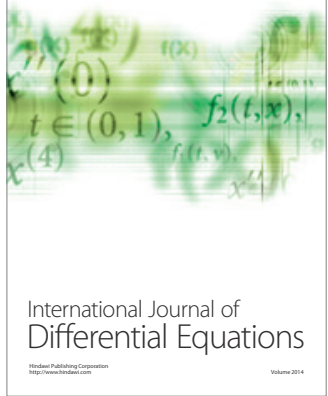
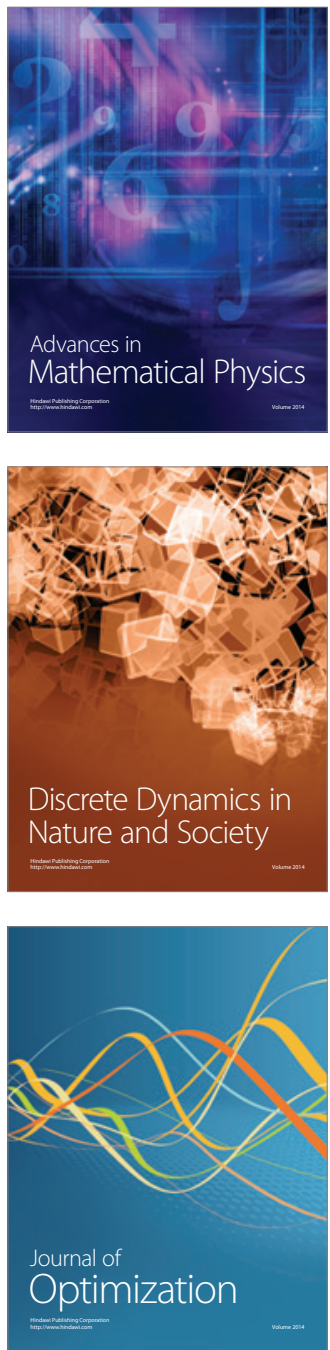